\title{
A posteriori error estimate in quantities of interest for the finite element heterogeneous multiscale method
}

\author{
A. Abdulle and A. Nonnenmacher *
}

October 12, 2012

\begin{abstract}
We present an a posteriori error analysis in quantities of interest for elliptic homogenization problems discretized by the finite element heterogeneous multiscale method. The multiscale method is based on a macro-to-micro formulation, where the macroscopic physical problem is discretized in a macroscopic finite element space and the missing macroscopic data is recovered on-the-fly using the solutions of corresponding microscopic problems. We propose a new framework that allows to follow the concept of the (single-scale) dual-weighted residual method at the macroscopic level in order to derive a posteriori error estimates in quantities of interests for multiscale problems. Local error indicators, derived in the macroscopic domain, can be used for adaptive goal-oriented mesh refinement. These error indicators rely only on available macroscopic and microscopic solutions. We further provide a detailed analysis of the data approximation error, including the quadrature errors. Numerical experiments confirm the efficiency of the adaptive method and the effectivity of our error estimates in the quantities of interest.
\end{abstract}

Keywords. multiscale problems, elliptic problems adaptive FEM, quantity of interest, homogenization, heterogeneous multiscale method

AMS 65N30, 65M60, 74Q05, 74Q20

\section{Introduction}

Multiscale models are nowadays found in many areas of science and engineering. They are for example crucial for the adequate simulation of groundwater pollution through infiltration of a fluid in a porous medium, or for finding the effective properties of composite materials important for various engineering applications. Multiscale models are also increasingly used in medicine for example to find the mechanical properties of heterogeneous tissues such as bones that are important to understand failure or diseases.

For such problems standard numerical approximation only converges if we can resolve the finest length scale (the microscopic length scale) of the multiscale problem. If this microscopic length scale is small in comparison to the macroscopic length scale of the multiscale problem, then the discrete approximation leads to a problem with very large degrees of freedom (DOF) and the computational complexity becomes often overwhelming. Simply ignoring the fine-scale microscopic structure leads to numerical results that do not appropriately reflect the true physical problem, as the microscopic structure significantly influences the macroscopic behavior.

In recent years there has therefore been a considerable effort put into the design of multiscale methods for elliptic PDEs that take into account the scale separation between macroscopic and microscopic models. Babuška and Osborn [1] developed the pioneering work for multiscale FEM for elliptic problems using multiscale basis functions. We further mention the multiscale finite element method (MsFEM) developed by Hou et al. 22 (see also the book by Efendiev and Hou [3]), the two-scale FEM proposed by Matache, Babuška and Schwab 4, the variational multiscale method by Hughes et al. [5], and the sparse FEM introduced by Hoang and Schwab [6]. In this work we use the framework of the heterogeneous multiscale method (HMM) proposed by E and Engquist [7, 8. In the HMM framework, one assumes that a macroscopic description of the multiscale problem exists, even though it may not be known explicitly. This macroscopic problem is solved on a coarse mesh using a macroscopic FEM. As the data of the effective problem (e.g., the conductivity tensor) is not known it is reconstructed on-the-fly by solving

\footnotetext{
*ANMC, Mathematics Section, cole Polytechnique Fdrale de Lausanne, 1015 Lausanne, Switzerland, \{assyr.abdulle,achim.nonnenmacher\}@epfl.ch.
} 
microscopic problems on sampling domains located within the respective macroscopic elements. The HMM is related to numerical homogenization methods, where the fine scales are averaged out in order to obtain effective (homogenized) equations. For elliptic problems, a semi-discrete a priori analysis for the FE-HMM was given in [9, 10] and a fully discrete analysis was obtained in [1, 12, 13. Furthermore, discontinuous Galerkin FE-HMM was developed in [14, 15] and problems in elasticity were studied in [16. See also 17 for a thorough overview.

A crucial issue when using a numerical scheme is that of estimating the error or the reliability of an actual computed solution. Such estimation are usually obtained from a posteriori error analysis. The goal of a posteriori error estimates is not only to offer a criterion that indicates whether a certain prescribed accuracy is met, but also to give local error indicators, which can be used to drive an adaptive mesh refinement that equi-distributes the approximation error among the elements and therefore minimizes the total computational effort. Since the pioneering work of Babuška and Rheinboldt [18, numerous results on this research topic have been obtained and a vast literature on a posteriori error analysis for (single scale) elliptic PDEs is nowadays available (see for example [19, Verfürth [20] and Babuška and Strouboulis 21 and the references therein). In practical applications, scientists and engineers are however often more interested in errors in a certain quantity of interest that is needed for an engineering design decision than in errors in the energy norm. Such quantities of interests can for example be an average heat flux through a certain boundary, local averages of the solution in a particular region of interest, etc. In turn, goal-oriented error estimates in quantities of interest have been developed in the late 1990s. The general approach is as follows. Assuming that the quantity of interest can be represented by a linear bounded functional $J: V \rightarrow \mathbb{R}$ of the solution, the task is then to estimate $J\left(u-u^{H}\right)$, where $u$ is the exact solution of the considered PDE and $u^{H}$ is its FE approximation. Expressing $J\left(u-u^{H}\right)$ in terms of an exact representation of local error estimators and higher order terms (using so-called primal and dual problems), one can implement the following cycle

$$
\text { Solve } \rightarrow \text { Estimate } \rightarrow \text { Mark } \rightarrow \text { Refine }
$$

using suitable local error estimators, until the error $\left|J\left(u-u^{H}\right)\right|$ in the estimate step is smaller than a predefined tolerance. Among the different strategies for error estimates in quantitates of interest, we mention the work of Prudhomme and Oden [22, 23, the review of Giles and Süli 24], Bangerth and Rannacher [25] and Becker and Rannacher [26. The latter developed the so-called dual-weighted residual (DWR) method. The reliability of the DWR method was further investigated by Nochetto et al. 27. and Ainsworth and Rankin [28. Finally, a goal-oriented method aiming at adaptively controlling various models of a multiscale problem has been developed by Oden and co-worker 29].

We briefly review a few contributions related to adaptivity for multiscale problems. We first mention the approach based on the variational multiscale method by Hughes et al. [30, 5, which decomposes the solution into a coarse-scale and a fine-scale part. The solution of the fine-scale equation is formulated in dependence of the residual of the macro solution. A posteriori error estimates in the energy norm (upper bounds) were derived by Larson and Målqvist in [31. Finally we mention the general framework for adaptive methods developed Nolen, Papanicolaou and Pironneau 32. Algorithms and analysis for residual-based estimators (which estimates the error of the energy norm) for the FE-HMM have been obtained by Ohlberger 33] for linear elliptic problems and by Henning and Ohlberger for monotone operators [34. These a posteriori error estimates are based on a reformulation of the FE-HMM in a twoscale framework [35]. First a posteriori error estimates for the FE-HMM in the physical energy-norm have been obtained in [36, 37. The improvement in computational efficiency that is obtained by introducing adaptivity is significant as solutions of the problems on the microscopic scale (the main computational cost of the FE-HMM) may be re-used in an adaptive mesh refinement strategy [37.

The modeling and analysis of a posteriori error estimates in quantities of interest have not yet been developed for numerical homogenization methods to the best of the authors' knowledge. In this work we propose goal-oriented a posteriori error estimates for the FE-HMM. Although our strategy follows the DWR method for single-scale problems, several non-trivial issues need to be addressed for its extension to multiscale methods as

- variational crimes are inherent to the formulation of the macroscopic bilinear form of the FE-HMM that is based on numerical quadrature;

- the FE-HMM is not consistent (due to the use of numerical quadrature and approximated effective data);

- the interior and jump residuals, ingredients of the local error estimator in the DWR method are 
not available in our multiscale strategy (as the data of the effective problem are not available) and need to be modeled based solely on the micro solutions available in the sampling domains.

Variational crimes and non-consistency that seem not to have been addressed for a posteriori error estimates in quantities of interest, already for single scale problems, will be discussed here. We will derive an exact representation for the error in a quantity of interest in term of error estimators and data approximation terms for general multiscale elliptic problems without any specific assumption on the small scale structure (e.g., such as periodicity). For locally periodic tensor (with possible discontinuities across macro elements), we will show that our approach is consistent with the DWR method of Becker and Rannacher [26 in the following sense: when the micro solver is exact we will recover the estimator and similar data approximation terms as in the classic DWR method. We notice that most of the multiscale methods developed for general multiscale problems are usually analyzed in the periodic setting as this allows to rely on quantitative bounds for the effective solution and so-called correctors (see [3, 8] and the references therein). The DWR method developed in [26 relies on the assumption that the data approximation terms (so-called higher order term) are negligible. This was later questioned by Nochetto et al. 27] that showed that neglecting the higher order terms can cause a severe underestimation of the error and suggested safeguarded DWR method. Finally Ainsworth and Rankin 28 developed guaranteed and fully computable bounds on the error in quantities of interest. We close this introduction by mentioning that including the recent finding of Ainsworth and Rankin 28. in our multiscale DWR method is of great interest. As our contribution constitutes the first DWR method for multiscale problems we concentrate here on the issues mentioned above (variational crimes, non-consistency, modeling of multiscale interior and jump residuals) and we leave the investigation of the data approximation term in the spirit of 28 for future research.

The outline of this article is as follows. In Section 2 we briefly review the FE-HMM and the residualbased adaptive FE-HMM. In Section 3 we present the main results for the goal-oriented adaptive FE-HMM that are proved in Section 4. We show the efficiency and reliability of the method in various numerical experiments in Section 5. A summary of our main findings and outlook are given in the conclusion in Section 6

Notation. In what follows, $C>0$ denotes a generic constant, independent of $\varepsilon$, whose value can change at any occurrence but depends only on the quantities which are indicated explicitely. For $r=$ $\left(r_{1}, \ldots, r_{d}\right) \in \mathbb{N}^{d}$, we denote $|r|=r_{1}+\ldots+r_{d}, D^{r}=\partial_{1}^{r_{1}} \ldots \partial_{d}^{r_{d}}$. We will consider the usual Sobolev space $H^{1}(\Omega)=\left\{u \in L^{2}(\Omega) ; D^{r} u \in L^{2}(\Omega),|r| \leq 1\right\}$, with norm $\|u\|_{H^{1}(\Omega)}=\left(\sum_{|r| \leq 1}\left\|D^{r} u\right\|_{L^{2}(\Omega)}^{2}\right)^{1 / 2}$. We will also consider $H_{0}^{1}(\Omega)$ the closure of $C_{0}^{\infty}(\Omega)$ for the $\|\cdot\|_{H^{1}(\Omega)}$ norm and the spaces $W^{l, \infty}(\Omega)=$ $\left\{u \in L^{\infty}(\Omega) ; D^{r} u \in L^{\infty}(\Omega),|r| \leq l\right\}$. For the unit cube $Y=(0,1)^{d}$, we will consider $W_{p e r}^{1}(Y)=$ $\left\{v \in H_{\text {per }}^{1}(Y) ; \int_{Y} v d x=0\right\}$, where $H_{\text {per }}^{1}(Y)$ is defined as the closure of $\mathcal{C}_{p e r}^{\infty}(Y)$ (the subset of $\mathcal{C}^{\infty}\left(\mathbb{R}^{d}\right)$ of periodic functions in $Y$ ) in the $H^{1}$ norm. Finally, we will use the Frobenius matrix norm $\|a\|_{F}:=$ $\sqrt{\sum_{i} \sum_{j}\left|a_{i j}\right|^{2}}$.

\section{Model problem and homogenization}

We consider the elliptic problem with oscillatory coefficients in a domain $\Omega \subset \mathbb{R}^{d}$

$$
\begin{aligned}
-\nabla \cdot\left(a^{\varepsilon} \nabla u^{\varepsilon}\right)=f & \text { in } \Omega, \\
u^{\varepsilon}=g_{D} & \text { on } \partial \Omega,
\end{aligned}
$$

where we impose Dirichlet boundary conditions $g_{D} \in L^{2}(\partial \Omega)$ and $f \in L^{2}(\Omega)$. We emphasize the multiscale nature of the problem by using the superscript $\varepsilon$. We assume that $a^{\varepsilon}$ is symmetric, satisfies $a^{\varepsilon}(x) \in\left(L^{\infty}(\Omega)\right)^{d \times d}$ and is uniformly elliptic and bounded, i.e.,

$$
\exists \lambda, \Lambda>0 \text { such that } \lambda|\xi|^{2} \leq a^{\varepsilon}(x) \xi \cdot \xi \leq \Lambda|\xi|^{2}, \forall \xi \in \mathbb{R}^{d} \text { and } \forall \varepsilon .
$$

An application of the Lax-Milgram theorem ensures the existence of a family of solutions $\left\{u^{\varepsilon}\right\}$ that is bounded in $H_{D}^{1}(\Omega)$. From homogenization theory we know that there exists a symmetric tensor $a^{0}(x)$ and a subsequence of $\left\{u^{\varepsilon}\right\}$ that weakly converges to the so-called homogenized solution $u^{0} \in H_{D}^{1}(\Omega)$ (we refer the reader to [38, 39] and references therein). The proof relies on G-convergence [40] (or Hconvergence [41]) and no further assumptions on the spatial structure of $a^{\varepsilon}(x)$ are needed for the weak 
convergence of a subsequence of $\left\{u^{\varepsilon}\right\}$. The homogenized solution $u^{0}$ solves a homogenized problem given by

$$
\begin{aligned}
-\nabla \cdot\left(a^{0} \nabla u^{0}\right)=f & \text { in } \Omega, \\
u^{0}=g_{D} & \text { on } \partial \Omega_{D},
\end{aligned}
$$

where the homogenized tensor $a^{0}(x)$ is again symmetric and uniformly elliptic and bounded. Under the assumption that $a^{\varepsilon}(x)=a(x, x / \varepsilon)$ is periodic in its second argument, it can be shown that the whole sequence $\left\{u^{\varepsilon}\right\}$ weakly converges to $u^{0} \in H_{D}^{1}(\Omega)$. In this case, we can also express the homogenized tensor $a^{0}(x)$ at a point $x \in \Omega$ in terms of solutions of $d$ bounday value problems, the so-called cell problems. We remark that in general there are infinitely many cell problems to solve. For more general tensors $a^{\varepsilon}$, an explicit equation to compute the homogenized tensor at a point $x \in \Omega$ is not available. We therefore follow a numerical strategy, here the Finite Element Heterogeneous Multiscale Method (FE-HMM), in order to compute a numerically homogenized solution.

\subsection{The Finite Element Heterogeneous Multiscale Method (FE-HMM)}

We use the finite element heterogeneous multiscale method (FE-HMM) to obtain an approximation $u^{H M M}$ to the homogenized (coarse) solution $u^{0}$ of $(3)$ without computing the homogenized tensor $a^{0}(x)$ explicitly. We only need the oscillatory data $a^{\varepsilon}(x)$ to be given on sampling domains. The basic framework of the FE-HMM consists in a coupling of a macro problem and a micro problem, described below. For simplicity of presentation we will take zero Dirichlet boundary conditions in (1) and set $g_{D}=0$.

Macro finite element space. We consider

$$
V^{p}\left(\Omega, \mathcal{T}_{H}\right)=\left\{v^{H} \in H_{0}^{1}(\Omega) ;\left.v^{H}\right|_{K} \in \mathcal{P}^{p}(K), \forall K \in \mathcal{T}_{H}\right\}
$$

with macro elements $K \in \mathcal{T}_{H}$, where $\mathcal{T}_{H}$ is assumed to be shape regular. For simplicity, we only consider simplicial (triangular, tetrahedral) elements, but emphasize that other elements could also be used. Here $\mathcal{P}^{p}$ is the space of piecewise polynomials on the element $K$ of total degree $p$, and $H$ is the size of the macro triangulation (notice that $H$ can be much larger than $\varepsilon$ ).

Quadrature formulas. We consider for each macro element $K \in \mathcal{T}_{H}$ a $C^{1}$-diffeomorphism $F_{K}$ such that $K=F_{K}(\hat{K})$, where $\hat{K}$ is a simplicial reference element. We consider a given quadrature formula (QF) $\left\{\hat{x}_{\ell}, \hat{\omega}_{\ell}\right\}_{\ell=1}^{\mathcal{L}}$ on the reference element $\hat{K}$; the corresponding integration points on an element $K \in \mathcal{T}_{H}$ are given by $x_{K_{\ell}}=F_{K}\left(\hat{x}_{\ell}\right), \ell=1, \ldots, \mathcal{L}$ and the corresponding quadrature weights on $K$ are given by $\omega_{K_{\ell}}=\hat{\omega}_{\ell}\left|\operatorname{det}\left(\partial F_{K}\right)\right|, \ell=1, \ldots, \mathcal{L}$. We make the following assumptions on the quadrature formulas (see 42]):

(Q) $\hat{\omega}_{\ell}>0, \ell=1, \ldots, \mathcal{L}, \quad \int_{\hat{K}} \hat{p}(\hat{x}) d \hat{x}=\sum_{\ell=1}^{\mathcal{L}} \hat{\omega}_{\ell} \hat{p}\left(\hat{x}_{\ell}\right), \forall \hat{p}(\hat{x}) \in \mathcal{P}^{\sigma}(\hat{K})$, where $\sigma=\max (2 p-2, p)$.

Remark 1. We notice here that the condition $\sigma=1$ for $\mathcal{P}^{1}$ is needed in the FE-HMM for optimal macro $L^{2}$ bounds (see [17]). Such bounds will not be discussed here and in the sequel assumption (Q2) can be taken with $\sigma=2 p-2$.

Macro bilinear form. For a discretization in the macro FE space (4) we define the FE-HMM bilinear form with $v^{H}, w^{H} \in V_{D}^{p}\left(\Omega, \mathcal{T}_{H}\right)$ as

$$
B_{H M M}\left(v^{H}, w^{H}\right)=\sum_{K \in \mathcal{T}_{H}} \sum_{\ell=1}^{\mathcal{L}} \frac{\omega_{K_{\ell}}}{\left|K_{\delta_{\ell}}\right|} \int_{K_{\delta_{\ell}}} a^{\varepsilon}(x) \nabla v_{K_{\delta_{\ell}}}^{h} \cdot \nabla w_{K_{\delta_{\ell}}}^{h} d x
$$

where $v_{K_{\ell}}^{h}, w_{K_{\ell}}^{h}$ are appropriate micro functions defined on sampling domains $K_{\delta_{\ell}}$ (see below) and the factor $\left|K_{\delta_{\ell}}\right|$ gives the appropriate weight for the contribution of the integrals defined on $K_{\delta_{\ell}}$ instead of $K$. The FE-HMM bilinear form (5) is based by construction on numerical quadrature. Therefore, in our a posteriori analysis, we need to specially take care of the corresponding variational crimes.

Micro functions. For every macro element $K$ we compute the solutions $v_{K_{\ell}}^{h}$ (and $w_{K_{\ell}}^{h}$ ) of micro problems on sampling domains $K_{\delta_{\ell}}, \quad \ell=1, \ldots, \mathcal{L}$ within $K$. The contributions to the macro stiffness matrix are constructed from the micro functions given as follows: find $v_{K_{\ell}}^{h}$ such that $\left(v_{K_{\ell}}^{h}-v_{\mathrm{lin}, K_{\ell}}^{H}\right) \in S^{q}\left(K_{\delta_{\ell}}, \mathcal{T}_{h}\right)$ and

$$
\int_{K_{\delta_{\ell}}} a^{\varepsilon}(x) \nabla v_{K_{\ell}}^{h} \cdot \nabla z^{h} d x=0 \quad \forall z^{h} \in S^{q}\left(K_{\delta_{\ell}}, \mathcal{T}_{h}\right),
$$


where

$$
v_{\operatorname{lin}, K_{\ell}}^{H}(x)=v^{H}\left(x_{K_{\ell}}\right)+\left(x-x_{K_{\ell}}\right) \cdot \nabla v^{H}\left(x_{K_{\ell}}\right)
$$

is a linearization of the macro function $v^{H}$ at the integration point $x_{K_{\ell}}$ (see [10, 17, for details). The FE space is defined as

$$
S^{q}\left(K_{\delta_{\ell}}, \mathcal{T}_{h}\right)=\left\{z^{h} \in W\left(K_{\delta_{\ell}}\right) ;\left.z^{h}\right|_{T} \in \mathcal{P}^{q}(T), T \in \mathcal{T}_{h}\right\},
$$

where $W\left(K_{\delta_{\ell}}\right)$ determines the coupling condition or boundary conditions used for computing the micro functions $v_{K_{\ell}}^{h}\left(\right.$ or $\left.w_{K_{\ell}}^{h}\right)$.

Coupling micro and macro solvers. Several choices are possible for the coupling condition $\left(v_{K_{\ell}}^{h}-\right.$ $\left.v_{\operatorname{lin}, K_{\ell}}^{H}\right) \in S^{q}\left(K_{\delta_{\ell}}, \mathcal{T}_{h}\right)$ and we will consider $S^{q}\left(K_{\delta_{\ell}}, \mathcal{T}_{h}\right) \subset W\left(K_{\delta_{\ell}}\right)$ with

$$
W\left(K_{\delta_{\ell}}\right)=W_{p e r}^{1}\left(K_{\delta_{\ell}}\right)
$$

for the periodic coupling and

$$
W\left(K_{\delta_{\ell}}\right)=H_{0}^{1}\left(K_{\delta_{\ell}}\right)
$$

for the Dirichlet coupling.

Variational problem. The macro solution of the FE-HMM is defined by the following variational problem: find $u^{H M M} \in V^{p}\left(\Omega, \mathcal{T}_{H}\right)$ such that

$$
B_{H M M}\left(u^{H M M}, v^{H}\right)=\int_{\Omega} f v^{H} d x \quad \forall v^{H} \in V^{p}\left(\Omega, \mathcal{T}_{H}\right) .
$$

The main goal of the FE-HMM is to find a solution $u^{H M M}$ that converges to the homogenized solution $u^{0}$. Remember that the FE-HMM also depends on a micro mesh, thus $h$ going to zero is also necessary for convergence (see below). It can be shown that the FE-HMM bilinear form (5) is elliptic and bounded, i.e., it satisfies

$$
B_{H M M}\left(v^{H}, v^{H}\right) \geq C\left\|v^{H}\right\|_{H^{1}(\Omega)}^{2}, \quad\left|B_{H M M}\left(v^{H}, w^{H}\right)\right| \leq C\left\|v^{H}\right\|_{H^{1}(\Omega)}\left\|w^{H}\right\|_{H^{1}(\Omega)},
$$

for all $v^{H}, w^{H} \in V^{p}\left(\Omega, \mathcal{T}_{H}\right)$ with a constant $C$ that only depends on the quadrature formula, the domain $\Omega$, the constant in (2). From the Lax-Milgram Theorem, existence and uniqueness of the solution $u^{H M M}$ of problem (11) immediately follows (we refer to [11, 17, 10] for details).

A priori estimates. The sources of errors for the FE-HMM approximation to the homogenized solution can be decomposed as follows [11, 17, 43, 10,

$$
\left\|u^{0}-u^{H M M}\right\| \leq\left\|u^{0}-u^{0, H}\right\|+\left\|u^{0, H}-\bar{u}^{H M M}\right\|+\left\|\bar{u}^{H M M}-u^{H M M}\right\|,
$$

where the first term on the right-hand side of the above inequality represents the macro error, the second term represents the modeling error and the last term represents the micro error. Here, $u^{0}$ is the solution of (3), $u^{H M M}$ is the FE-HMM solution of (11), $u^{0, H}$ is the FEM solution of the homogenized problem with numerical quadrature of (3) in the space $V^{p}\left(\Omega, \mathcal{T}_{H}\right)$ (using the same QF as for the FE-HMM) and $\bar{u}^{H M M}$ is the FE-HMM solution of (11) with exact micro functions (in $W\left(K_{\delta_{\ell}}\right)$ ). The general form of the a priori error estimate in the $H^{1}$-norm under the appropriate assumptions of $a^{\varepsilon}(x)$ is

$$
\left\|u^{0}-u^{H M M}\right\|_{H^{1}(\Omega)} \leq C\left(H^{p}+r_{M I C}+r_{M O D}\right),
$$

where $r_{M I C}, r_{M O D}$ are further described below. For the macro error $C H^{p}$, appropriate regularity of the homogenized tensor and homogenized solution is needed. For the micro error appropriate regularity of the tensor $a^{\varepsilon}$ is needed. We briefly recall the assumption on the micro problem as this will be needed in this paper. Consider $\psi_{K_{\delta_{\ell}}}^{i, h}$, the solution of the following micro problem on the sampling domain $K_{\delta_{\ell}}$

$$
\int_{K_{\delta_{\ell}}} a^{\varepsilon}(x) \nabla \psi_{K_{\delta_{\ell}}}^{i, h} \cdot \nabla z^{h} d x=-\int_{K_{\delta_{\ell}}} a^{\varepsilon}(x) \mathbf{e}_{i} \cdot \nabla z^{h} d x \quad \forall z^{h} \in S^{q}\left(K_{\delta_{\ell}}, \mathcal{T}_{h}\right),
$$

where $\mathbf{e}_{i}, i=1, \ldots, d$, denote the canonical basis of $\mathbb{R}^{d}$.

We define on every macro quadrature point a numerically homogenized tensor denoted as $a_{K}^{0}\left(x_{K_{\ell}}\right)$,

$$
a_{K}^{0}\left(x_{K_{\ell}}\right)=\frac{1}{\left|K_{\delta_{\ell}}\right|} \int_{K_{\delta_{\ell}}} a^{\varepsilon}(x)\left(I+J_{\psi_{K_{\delta_{\ell}}}^{h}}^{T}(x)\right) d x,
$$


where $J_{\psi_{K_{\delta_{\ell}}}^{h}(x)}$ is a $d \times d$ matrix whose entries are given by $\left(J_{\psi_{K_{\delta_{\ell}}}^{h}(x)}\right)_{i j}=\left(\partial \psi_{K_{\delta_{\ell}}}^{i, h}\right) /\left(\partial x_{j}\right)$. Next, we introduce the tensor $\bar{a}_{K}^{0}\left(x_{K_{\ell}}\right)$, which is defined as $a_{K}^{0}\left(x_{K_{\ell}}\right)$ given in $(15)$, but where the corresponding functions $\psi_{K_{\delta_{\ell}}}^{i}$ are found in the exact Sobolev space $W\left(K_{\delta_{\ell}}\right)$ instead of its FE approximation $S^{q}\left(K_{\delta_{\ell}}, \mathcal{T}_{h}\right)$. Finally we will also consider for a sufficiently regular homogenized problem (see (3)), the homogenized tensor $a^{0}\left(x_{K_{\ell}}\right)$ evaluated at the integration point $x_{K_{\ell}}$. We can then express $r_{M I C}$ and $r_{M O D}$ in terms of the above tensors as follows [43, 15, 8]

$$
\begin{aligned}
r_{M I C} & :=\sup _{K \in \mathcal{T}_{H}} \max _{1 \leq \ell \leq \mathcal{L}}\left\|\bar{a}_{K}^{0}\left(x_{K_{\ell}}\right)-a_{K}^{0}\left(x_{K_{\ell}}\right)\right\|_{F}, \\
r_{M O D} & :=\sup _{K \in \mathcal{T}_{H}} \max _{1 \leq \ell \leq \mathcal{L}}\left\|a^{0}\left(x_{K_{\ell}}\right)-\bar{a}_{K}^{0}\left(x_{K_{\ell}}\right)\right\|_{F} .
\end{aligned}
$$

To estimate the micro error, we will need the following regularity assumption

(H1) Given $q \in \mathbb{N}$, the cell functions $\psi_{K_{\ell}}^{i}$ satisfy

$$
\left|\psi_{K_{\ell}}^{i}\right|_{H^{q+1}\left(K_{\delta_{\ell}}\right)} \leq C \varepsilon^{-q} \sqrt{\left|K_{\delta_{\ell}}\right|}
$$

with $C$ independent of $i=1 \ldots d$, $\varepsilon$, the quadrature point $x_{K_{\ell}}$ and the domain $K_{\delta_{\ell}}$.

We note that if $\left.a_{i j}^{\varepsilon}\right|_{K} \in W^{1, \infty}(K) \forall K \in \mathcal{T}_{H}$ and $\left|a_{i j}^{\varepsilon}\right|_{W^{1, \infty}(K)} \leq C \varepsilon^{-1}$, then classical $H^{2}$ regularity results ([44, Chap. 2.6]) imply that $\left|\psi_{K_{\ell}}^{i}\right|_{H^{2}\left(K_{\delta_{\ell}}\right)} \leq C \varepsilon^{-1} \sqrt{\left|K_{\delta_{\ell}}\right|}$ when Dirichlet boundary conditions $W\left(K_{\delta_{\ell}}\right)=H_{0}^{1}\left(K_{\delta_{\ell}}\right)$ are used. If $a(x, x / \varepsilon)=a(x, y)$ is periodic in the $\mathrm{y}$ variable, then we can also use periodic boundary conditions $W\left(K_{\delta_{\ell}}\right)=H_{\text {per }}^{1}\left(K_{\delta_{\ell}}\right)$, and higher regularity for $\psi_{K_{\ell}}^{i}$ can be shown, provided $a(x, \cdot)$ is smooth enough and $\delta / \varepsilon \in \mathbb{N}$ (see [45, Chap. 3]). We refer to [17, 43] for details.

Under assumption (H1), the following estimate is valid [11, 43, 15]

$$
r_{M I C} \leq C\left(\frac{h}{\varepsilon}\right)^{2 q}
$$

where $C$ is independent of $H, h$. Finally to estimate the modeling error, structure assumptions on the coefficients $a^{\varepsilon}$ are needed.

$$
\begin{aligned}
& a^{\varepsilon}(x)=a(x, x / \varepsilon)=a(x, y) \quad \text { is } Y \text {-periodic in } y, \\
& a_{i j}(x, y) \in \mathcal{C}\left(\bar{\Omega} ; W_{\text {per }}^{1, \infty}(Y)\right), \text { for all } i, j=1, \ldots, d .
\end{aligned}
$$

Now if (H2) holds and if [6] is solved in $S^{q}\left(K_{\delta_{\ell}}, \mathcal{T}_{h}\right) \subset H_{0}^{1}\left(K_{\delta_{\ell}}\right)$ with $\delta>\varepsilon$, then [10]

$$
r_{M O D} \leq C\left(\frac{\varepsilon}{\delta}+\delta\right)
$$

where $C$ is independent of $H$ and $h$. If furthermore the micro problems (6) are solved in $S^{q}\left(K_{\delta_{\ell}}, \mathcal{T}_{h}\right)$ $\subset W_{\text {per }}^{1}\left(K_{\delta_{\ell}}\right)$ with $\delta / \varepsilon \in \mathbb{N}$, the slow variable of the tensor $a(x, x / \varepsilon)$ is collocated at the quadrature points $x_{K_{\ell}}$, i.e., $a\left(x_{K_{\ell}}, x / \varepsilon\right)$ in the problem (6) and in the bilinear form (5) then it can be shown that 9]

$$
r_{M O D}=0
$$

\section{Goal-oriented error estimation for the FE-HMM}

In this Section we developed goal oriented macroscopic error estimators to drive a mesh refinement towards the approximation of a given quantity of interest. Suppose that we are interested in a quantity $J\left(u^{0}\right)$, where $J$ denotes a linear bounded functional and $u^{0}$ is the homogenized solution. The question is now: is it possible to refine the numerical solution of the FE-HMM, to have an approximation of the quantity of interest $J\left(u_{H}\right) \simeq J\left(u^{0}\right)$ ?

To answer this question we will use the framework of the dual-weighted residual (DWR) method that relies on a primal and a dual problem to extract the solution in the quantity of interest. Localized macroscopic refinement indicators will be designed and used to drive an adaptive mesh refinement. 


\subsection{Single-scale DWR FEM}

We start by recalling the single scale DWR method. The primal problem for the homogenized solution $u^{0}$ is given by $(3)$, which reads in weak form

$$
B_{0}\left(u^{0}, v\right)=\int_{\Omega} f v d x \quad \forall v \in H_{0}^{1}(\Omega) .
$$

Let $u^{H} \in V^{p}\left(\Omega, \mathcal{T}_{H}\right)$ be the FEM solution (without quadrature) of

$$
B_{0}\left(u^{H}, v^{H}\right)=\int_{\Omega} f v^{H} d x \quad \forall v^{H} \in V^{p}\left(\Omega, \mathcal{T}_{H}\right) .
$$

By defining the dual solution $z^{0} \in H_{0}^{1}(\Omega)$ given by

$$
B_{0}\left(\varphi, z^{0}\right)=J(\varphi) \quad \forall \varphi \in H_{0}^{1}(\Omega),
$$

we obtain that

$$
J\left(u^{0}-u^{H}\right)=B_{0}\left(u^{0}-u^{H}, z^{0}\right) .
$$

As $z^{0} \in H_{0}^{1}(\Omega)$ however is not available, we compute an approximation $z^{\mathcal{H}} \in V_{\mathscr{H}}$, where $V_{\mathcal{H}}$ is yet to be determined. Then we have that

$$
J\left(u^{0}-u^{H}\right)=B_{0}\left(u^{0}-u^{H}, z^{0}\right)=\underbrace{B_{0}\left(u^{0}-u^{H}, z^{\mathcal{H}}\right)}_{=\eta_{H}(\Omega)}-\underbrace{B_{0}\left(u^{0}-u^{H}, z^{\mathcal{H}}-z^{0}\right)}_{=\xi_{H}(\Omega)},
$$

where $\eta_{H}(\Omega)$ is the error estimator, $\xi_{H}(\Omega)$ is the data approximation error. We note that one can further introduce an arbitrary function $\psi^{H} \in V^{p}\left(\Omega, \mathcal{T}_{H}\right)$ in the right-hand side of (25), i.e., replace it with $B_{0}\left(u^{0}-u^{H}, z^{\mathcal{H}}-\psi^{H}\right)+B_{0}\left(u^{0}-u^{H}, z^{\mathcal{H}}-z^{0}\right)$ due to the Galerkin orthogonality. This freedom can be used to try to increase the accuracy of the estimator or reduce the computational cost of the error estimator (see [25]). As mentioned earlier, in our multiscale framework, Galerkin orthogonality does not hold and we will suppose that $\psi^{H}=0$.

We can further decompose the error estimator as

$$
\eta_{H}(\Omega)=\sum_{K \in \mathcal{T}_{H}} \eta_{H}(K)=\int_{K} R_{I, H}(x) z^{\mathcal{H}} d x+\int_{\partial K} R_{J, H}(x) z^{\mathcal{H}} d s,
$$

where the interior and jump residual are given by

$$
\left.R_{I, H}\right|_{K}:=f^{H}+\nabla \cdot\left(a^{0} \nabla u^{H}\right),\left.\quad R_{J, H}\right|_{K}:=-\frac{1}{2} \llbracket a^{0} \nabla u^{H} \rrbracket_{e} .
$$

The flux term $\llbracket a^{0} \nabla u^{H} \rrbracket_{e}$ is defined by

$$
\llbracket a^{0} \nabla u^{H} \rrbracket_{e}:= \begin{cases}\left(a^{0} \nabla u^{H,+}-a^{0} \nabla u^{H,-}\right) \cdot n_{e} & \text { for } e \not \subset \partial \Omega, \\ 0 & \text { for } e \subset \partial \Omega,\end{cases}
$$

where $e$ is the interface between elements $K^{+}$and $K^{-}$with unit outward normals $n^{+}, n^{-}$, respectively and $n_{e}:=n^{+}$. We also used $f^{H}$, an approximation of $f$ in the space $\left\{g \in L^{2}(\Omega) ;\left.g\right|_{K} \in \mathcal{P}^{m}(K), \forall K \in \mathcal{T}_{H}\right\}$ for $m \geq 0$. Due to Galerkin orthogonality, simply replacing $z^{0}$ by its discrete solution $z^{H}$ in $V^{p}\left(\Omega, \mathcal{T}_{H}\right)$ and using the discrete primal solution $u^{H}$ will lead to $B_{0}\left(u^{0}-u^{H}, z^{H}\right)=0$. Therefore an other type of approximation $z^{\mathcal{H}}$ of $z^{0}$ is needed. Different strategies are discussed in the literature [25, 27, 46]

- Approximate the dual solution $z^{0}$ by the FEM solution $z^{\mathcal{H}}$ in a higher order polynomial space $V_{\mathcal{H}}:=V^{\tilde{p}}\left(\Omega, \mathcal{T}_{H}\right)$ with $\tilde{p}>p$.

- Approximate the dual solution $z^{0}$ by higher-order interpolation based on the solution $z^{H} \in V^{p}\left(\Omega, \mathcal{T}_{H}\right)$.

- Follow the $h$-approach and compute $z^{H}$ in $V_{\mathcal{H}}:=V^{p}\left(\Omega, \mathcal{T}_{\widetilde{H}}\right)$, where the triangulation $\mathcal{T}_{\widetilde{H}}$ is obtained from $\mathcal{T}_{H}$ by a suitable refinement of each element $K \in \mathcal{T}_{\widetilde{H}}$ into finer subelements.

As mentioned in the introduction, the computable quantity $\eta_{H}$ gives a realistic bound of the quantity of interest $J\left(u^{0}-u^{H}\right)$ only if the so-called higher order term $\xi_{H}$ is negligible. We refer to [27] and [28] were this issue is discussed and guaranteed and fully computable bounds on the error in quantities of interest are developed. As mentioned in the

Finally, we remark that variational crimes, introduced by e.g., choosing the dual solution in the finite dimensional space $V_{\mathcal{H}}$ and using a bilinear form based on numerical quadrature are typically disregarded in the literature, even for the single-scale DWR FEM. As the FE-HMM is based by construction on numerical quadrature, we will have to analyze the corresponding variational crimes. 


\subsection{DWR FE-HMM}

In order to develop a DWR FE-HMM, we have to address the issues of variational crimes (inherent to the the FE-HMM that is based on numerical quadrature), non-consistency of the FE-HMM solution and model appropriately interior and jump residuals based on the micro solutions available in the sampling domains.

Primal problem. We approximate $u^{0}$ by the FE-HMM solution $u^{H M M} \in V^{p}\left(\Omega, \mathcal{T}_{H}\right)$, where $u^{H M M}$ is the solution of

$$
B_{H M M}\left(u^{H M M}, v^{H}\right)=\int_{\Omega} f v^{H} d x \quad \forall v^{H} \in V^{p}\left(\Omega, \mathcal{T}_{H}\right),
$$

and where $B_{H M M}(\cdot, \cdot)$ refers to the FE-HMM bilinear form (5).

Dual problem. We choose the dual solution to be in the richer space

$$
V_{\mathcal{H}}:=V^{\tilde{p}}\left(\Omega, \mathcal{T}_{H}\right)
$$

the FE space of piecewise polynomials of degree $\tilde{p}>p$. The discretized dual problem (using the FE-HMM) is then

$$
B_{\mathcal{H M M}}\left(\varphi^{\mathcal{H}}, z^{\mathcal{H} \mathscr{M} \mathcal{M}}\right)=J\left(\varphi^{\mathscr{H}}\right) \quad \forall \varphi^{\mathscr{H}} \in V^{\tilde{p}}\left(\Omega, \mathcal{T}_{H}\right)
$$

where $B_{\mathscr{H} \mathfrak{M M}}(\cdot, \cdot)$ is the FE-HMM bilinear form (5) with a quadrature scheme satisfying $(\mathbf{Q})$ with $\sigma=$ $2 \tilde{p}-2$. We recall that we use the same family of triangulation $\mathcal{T}_{H}$ for the primal and dual problems. The micro and modeling errors defined in $(16), 17$ will be denoted by $r_{\mathcal{M I C}}, r_{\mathcal{M O D}}$ for the dual solution.

Remark 2. It is readily seen that the a priori estimates 13 for the macro error, (18) for the micro error and (19) for the modeling error are also valid (with $p$ replaced by $\tilde{p}$ ) for the dual solution $z^{\mathcal{H M M}}$ under similar assumptions as for the primal solution $u^{\mathrm{HMM}}$. In what follows, we assume that the same micro space $S^{q}\left(K_{\delta_{\ell}}, \mathcal{T}_{h}\right)$, the same family of micromeshes, and the same sampling domains size are used for the primal and dual solutions. Thus the estimates 180,19 (independent of the quadrature points) will be identical for $u^{\mathrm{HMM}}$ and $z^{\mathcal{H M M}}$.

\subsection{Main results}

Our goal is to find an exact representation of the error in the quantity of interest $J\left(u^{0}-u^{H M M}\right)$, where $J$ is a linear functional, $u^{0}$ the solution of the problem (3), and $u^{H M M}$ the solution of Problem (11). As the FE-HMM solution $u^{H M M}$ only relies on input data of the original multiscale problem (1), terms like $\nabla \cdot\left(a^{0} \nabla u^{H}\right)$ or $\llbracket \nabla \cdot\left(a^{0} \nabla u^{H}\right) \rrbracket_{e}$ (see $\left.(27)\right)$ involved in the interior and jump of the single-scale DWR method (for the homogenized problem) are not available and need to be modeled by expressions involving only the data of the problem (1).

\subsubsection{Jumps of multiscale fluxes for higher order polynomials.}

A key ingredient to model interior and jump residuals is the jump of multiscale fluxes first defined in 37, 15] in the context of discontinuous Galerkin FE-HMM. Consider the higher order macro FE space $V^{p}\left(\Omega, \mathcal{T}_{H}\right)$. Then, for a given $\mathrm{QF}\left\{x_{K_{\ell}}, \omega_{K_{\ell}}\right\}_{\ell=1}^{\mathcal{L}}$ that satisfies condition (Q) (see Section 2.1), we have the following relation between the order of the FE space $p$ and the number $\mathcal{L}$ of nodes of the QF (see for example [47, Section 1])

$$
\mathcal{L} \geq \frac{1}{2} p(p+1) \text { for } d=2, \quad \mathcal{L} \geq \frac{1}{6} p(p+1)(p+2) \text { for } d=3 .
$$

We consider quadrature formulae which minimize these inequalities in the following sense:

$$
\begin{cases}\mathcal{L}=\frac{1}{2} p(p+1) & \text { for } d=2 \\ \mathcal{L}=\frac{1}{6} p(p+1)(p+2) & \text { for } d=3\end{cases}
$$

For a QF satisfying (31), we consider the following interpolation problem: for $v \in C^{0}(K)$ find $\Pi_{v}(x) \in$ $\mathcal{P}^{p-1}(K)$ s.t.

$$
\Pi_{v}\left(x_{K_{\ell}}\right)=v\left(x_{K_{\ell}}\right), \quad \ell=1, \ldots, \mathcal{L},
$$

where the interpolation nodes $x_{K_{\ell}}$ are given by the quadrature nodes of the QF $\left\{x_{K_{\ell}}, \omega_{K_{\ell}}\right\}_{\ell=1}^{\mathcal{L}}$. We then have the following lemma which is easy to prove (see [48] for details) 
Lemma 3. Assume that (Q) and (31) hold. Then, the interpolation problem (32) has a unique solution.

We emphasize that condition (31) is satisfied by various well-known QF. (see for example [49]). We refer the reader to [4] for a general discussion on the condition (31) and to [50 for an overview of the construction of cubature formulae. For the FE-HMM, it is strongly advised to choose such a QF, as it minimizes the number of quadrature points and therefore the number of micro problems needed to be solved for each macro element.

To motivate the construction of the multiscale fluxes we recall the following result (see [15, Corollary $5])$. Let $v_{K_{\ell}}^{h}$ be the solution of (6) constrained by $v_{\text {lin }, K_{\ell}}^{H}(x)$, then

$$
\frac{1}{\left|K_{\delta_{\ell}}\right|} \int_{K_{\delta_{\ell}}} a^{\varepsilon}(x) \nabla v_{K_{\ell}}^{h} d x=a_{K}^{0}\left(x_{K_{\ell}}\right) \nabla v^{H}\left(x_{K_{\ell}}\right), \quad \ell=1, \ldots, \mathcal{L},
$$

where $a_{K}^{0}\left(x_{K_{\ell}}\right)$ is the numerically homogenized tensor defined in 15 for each sampling domain. On every macro element $K$ we then define the interpolating polynomial $\Pi_{a^{\varepsilon} \nabla v_{K}^{h}}(x)$ in $\left(\mathcal{P}^{p-1}(K)\right)^{d}$, which satisfies

$$
\Pi_{a^{\varepsilon} \nabla v_{K}^{h}}\left(x_{K_{\ell}}\right)=\frac{1}{\left|K_{\delta_{\ell} \mid}\right|} \int_{K_{\delta_{\ell}}} a^{\varepsilon}(x) \nabla v_{K_{\ell}}^{h} d x, \quad \ell=1, \ldots, \mathcal{L} .
$$

Assuming that (Q) and (31) hold, then the interpolation problem (34) is well defined by Lemma 3. We will refer to the interpolating polynomial $\Pi_{a^{\varepsilon} \nabla v_{K}^{h}}(x)$ as the higher order multiscale flux. Using (33) we see that

$$
\Pi_{a^{\varepsilon} \nabla v_{K}^{h}}\left(x_{K_{\ell}}\right)=a_{K}^{0}\left(x_{K_{\ell}}\right) \nabla v^{H}\left(x_{K_{\ell}}\right), \quad \ell=1, \ldots, \mathcal{L} .
$$

We further introduce for each interior interface $e$ of two elements $K^{+}$and $K^{-}$(with unit outward normals $n^{+}, n^{-}$, respectively) the following jump of higher order multiscale fluxes

$$
\llbracket \overline{\Pi_{a^{\varepsilon} \nabla v_{K}^{h}}} \rrbracket_{e}(s):= \begin{cases}\left(\Pi_{a^{\varepsilon} \nabla v_{K^{+}}^{h}}(s)-\Pi_{a^{\varepsilon} \nabla v_{K^{-}}^{h}}(s)\right) \cdot n_{e} & \text { for } e \not \subset \partial \Omega, \\ 0 & \text { for } e \subset \partial \Omega\end{cases}
$$

where the unit outward normal $n_{e}$ is chosen to be $n_{e}=n^{+}$.

Remark 4. When the simplicial macro FE space is poiecewise linear, $\mathcal{L}=1$ and we have only one quadrature point per macro element (36) can be defined as [15])

$$
\overline{\llbracket a^{\varepsilon} \nabla u_{K}^{h} \rrbracket} \rrbracket_{e}:=\left\{\begin{array}{cl}
\left(\frac{1}{\left|K_{\delta}^{+}\right|} \int_{K_{\delta}^{+}} a^{\varepsilon}(x) \nabla v_{K^{+}}^{h}(x) d x-\frac{1}{\left|K_{\delta}^{-}\right|} \int_{K_{\delta}^{-}} a^{\varepsilon}(x) \nabla v_{K^{-}}^{h}(x) d x\right) \cdot n_{e} & \text { for } e \not \subset \partial \Omega, \\
0 & \text { for } e \subset \partial \Omega .
\end{array}\right.
$$

\subsubsection{Exact error representation and error estimator for the DWR-FE-HMM}

Our first main result is an exact representation of the error in a quantity of interest that is valid without specific assumptions (e.g. as periodicity or stationarity) on the small scales $a^{\varepsilon}$ (recall that the FE-HMM relies solely on this problem). The proof of this DWR FE-HMM error representation theorem will be given in Section 4 .

Theorem 5 (Exact DWR FE-HMM error representation). Let $u^{0}$ be the solution of the problem (3), and $u^{\mathrm{HMM}}$ the solution of Problem (11). Let $J: H_{0}^{1}(\Omega) \rightarrow \mathbb{R}$ be a bounded linear functional and assume that $(\boldsymbol{Q})$ and (31) hold. Then the error in quantities of interest is given by the exact representation

$$
J\left(u^{0}-u^{\mathrm{HMM}}\right)=\sum_{K \in \mathcal{T}_{H}} \eta_{H}(K)+\xi_{H}(K),
$$

where

$$
\eta_{H}(K):=\int_{K} R_{I, H}(x) z^{\mathcal{H} \mathfrak{M M M}} d x+\int_{\partial K} R_{J, H}(x) z^{\mathcal{H} \mathfrak{M M}} d s
$$

and

$$
\left.R_{I, H}(x)\right|_{K}=f^{H}+\nabla \cdot\left(\Pi_{a^{\varepsilon} \nabla u_{K}^{h}}(x)\right),\left.\quad R_{J, H}(s)\right|_{e}=-\frac{1}{2} \llbracket \overline{\Pi_{a^{\varepsilon} \nabla u_{K}^{h}}} \rrbracket_{e}(s) .
$$


The data approximation error $\xi_{H}(K)$ is given by

$$
\begin{aligned}
\xi_{H}(K)= & \int_{K}\left(\Pi_{a^{\varepsilon} \nabla u_{K}^{h}}(x)-a^{0}(x) \nabla u^{\mathrm{HMM}}\right) \cdot \nabla z^{\mathcal{H} \mathfrak{M M}} d x \\
& +B_{0, K}\left(u^{0}-u^{\mathrm{HMM}}, z^{0}-z^{\mathcal{H M M}}\right) \\
& -\int_{K}\left(f^{H}-f\right) z^{\mathcal{H M M}} d x
\end{aligned}
$$

where the subscript ${ }_{K}$ in $B_{0, K}(\cdot, \cdot)$ indicates the restriction of $B_{0}(\cdot, \cdot)$ onto the element $K$ and where $f^{H}$ is an approximation of $f$ in the space $\left\{g \in L^{2}(\Omega) ;\left.g\right|_{K} \in \mathcal{P}^{m}(K), \forall K \in \mathcal{T}_{H}\right\}$ for $m \geq 0$.

The components of the data approximation error $(39)$ can be understood as follows: (39a) is the approximation error caused by using an inaccurate tensor (implicitly computed within the micro problem) and numerical quadrature instead of using the exact continuous $a^{0}(x)$ in the estimator; $39 \mathrm{~b}$ is the approximation error for using $z^{\mathcal{H M M}}$ instead of $z^{0}$; finally, $39 \mathrm{c}$ is the approximation error caused by approximating the right-hand side $f(x)$ in the estimator.

The DWR FE-HMM allows for cancelation of errors among elements; we therefore need to distinguish between the (global) signed error representation with local error estimators $\eta_{H}(K)$ (see Theorem 5 ), used to estimate the global error of the approximation with the unsigned local refinement indicators $\bar{\eta}_{H}(K)$

$$
\bar{\eta}_{H}(K):=\left|\int_{K} R_{I, H}(x)\left(z^{\mathcal{H} \mathcal{M M}}\right) d x+\int_{\partial K} R_{J, H}(s)\left(z^{\mathcal{H} \mathcal{M} \mathscr{M}}\right) d s\right|
$$

used to drive the adaptive mesh refinement procedure (see the Algorithm below). Obviously we have

$$
\left|J\left(u^{0}-u^{H M M}\right)\right| \leq \sum_{K \in \mathcal{T}_{H}} \bar{\eta}_{H}(K)+\left|\xi_{H}(K)\right|
$$

Remark 6. As the refinement indicators $\bar{\eta}_{H}(K)$ are always positive we cannot, in general, find the optimal mesh which would take error cancelation among different elements into account. We remark that finding the optimal mesh is also not possible for the single-scale DWR FEM. While being not optimal, the mesh found using positive refinement indicators still leads to good convergence rates. However, the convergence curves might be very jagged instead of being a straight line.

Our next main result (again proved in Section 4 gives an error bound on the data approximation error.

Theorem 7 (Error bound for data approximation error). Assume that (Q) holds with $\sigma=2 p-2$ (primal solution) and $\sigma=2 \tilde{p}-2$ (dual solution). Assume in addition that (31) holds for QF used for the primal

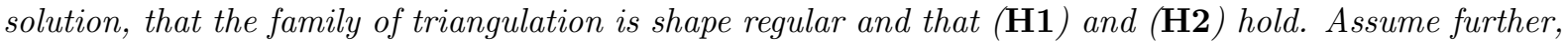
that $\left.a(\cdot, y)\right|_{K}$, is constant for any $K \in \mathcal{T}_{H}$. Then, the data approximation error $\xi_{H}(\Omega)$ can be estimated as follows

$$
\left|\xi_{H}(\Omega)\right| \leq C\left(H^{p+\tilde{p}}+\left(\frac{h}{\varepsilon}\right)^{2 q}+\left\|f-f^{H}\right\|_{L^{2}(K)}+r_{M O D}\right)
$$

where

$$
\begin{array}{ll}
r_{M O D}=0 & \text { if } S^{q}\left(K_{\delta_{\ell}}, \mathcal{T}_{h}\right) \subset W_{p e r}^{1}\left(K_{\delta_{\ell}}\right) \text { and } \frac{\delta}{\varepsilon} \in \mathbb{N}, \\
r_{M O D} \leq C\left(\varepsilon+\left(\frac{\varepsilon}{\delta}\right)\right) & \text { if } S^{q}\left(K_{\delta_{\ell}}, \mathcal{T}_{h}\right) \subset H_{0}^{1}\left(K_{\delta_{\ell}}\right)(\delta>\varepsilon) .
\end{array}
$$

and where the constants $C$ are independent of $H, h$ and $\varepsilon$.

Remark 3.1. We notice that the the additional assumption $\left.a(\cdot, y)\right|_{K}$, is constant is convenient to estimate the multiscale fluxes and can be removed for $p=1$. In that case, we obtain

$$
\left|\xi_{H}(\Omega)\right| \leq C\left(H+\left(\frac{h}{\varepsilon}\right)^{2 q}+\left\|f-f^{H}\right\|_{L^{2}(K)}+r_{M O D}\right) .
$$

We emphasize that the error estimator of the DWR-FE-HMM method exploits cancelation of errors over different elements and we therefore expect the above estimates to significantly overestimate the real data approximation error. 
Corollary 8 (Consistency with single scale DWR). In addition to the assumptions of Theorem 7 , assume that the micro problems (6) are solved exactly (i.e. in $W_{p e r}^{1}\left(K_{\delta_{\ell}}\right)$ and that $\delta / \varepsilon \in \mathbb{N}$, then

$$
\left.R_{I, H}\right|_{K}:=f+\nabla \cdot\left(a^{0} \nabla u^{H}\right),\left.\quad R_{J, H}\right|_{K}:=-(1 / 2) \llbracket a^{0} \nabla u^{H} \rrbracket_{e} .
$$

and the data approximation error $\xi_{H}(\Omega)$ can be estimated as

$$
\left|\xi_{H}(\Omega)\right| \leq C H^{p+\tilde{p}}+C\left\|f-f^{H}\right\|_{L^{2}(K)} .
$$

We see from this corollary, that we obtain exactly the same interior and jump residual than in the single-scale DWR method (see (27) ) for periodic problems (if no micro error due to the FE discretization of the problem (6) occur). The error bound for the data approximation term is also the same as in the classical DWR method. This corollary constitute the motivation for modeling the multiscale fluxes and defining the related interpolation polynomials.

We next give a detailed description of the adaptive DWR FE-HMM that consists of loops of the form

$$
\text { Solve } \rightarrow \text { Estimate } \rightarrow \text { Mark } \rightarrow \text { Refine. }
$$

The detailed step-by-step procedure is given below.

[Adaptive DWR-FE-HMM]

Solve. For the macro and micro meshes obtained by REFINE, compute the micro solutions (only for the refined macro elements) and the macro solutions $u^{\mathrm{HMM}}$ and $z^{\mathcal{H M M}}$ for the primal and the dual problem, respectively. Compute the micro function $\varphi_{K_{e}}^{h}$ solution of (6) constrained by the FE basis functions $\varphi^{H} \in V^{p}\left(\Omega, \mathcal{T}_{H}\right)$ and compute and store the higher-order multiscale fluxes $\Pi_{a^{\varepsilon} \nabla \varphi_{K}^{h}}\left(x_{K_{\ell}}\right)$.

Estimate. Reconstruct the full multiscale fluxes $\Pi_{a^{\varepsilon} \nabla u_{K}^{h}}(x)$ and jumps $\llbracket \overline{\Pi_{a^{\varepsilon} \nabla u_{K}^{h}}} \rrbracket_{e}(s)$ (once $u^{\mathrm{HMM}}$ is known). Estimate the error in the quantity of interest $J\left(u^{0}-u^{\mathrm{HMM}}\right)$ by computing the estimate $\sum_{K \in \mathcal{T}_{H}} \eta_{H}(K)$ (this estimate can serve as a stopping criterion using $\left|\sum_{K \in \mathcal{T}_{H}} \eta_{H}(K)\right|<t o l$, for a prescribed tolerance tol).

Mark. Mark the elements on a subset $\widetilde{\mathcal{T}}_{H}$ of $\mathcal{T}_{H}$ based on the refinement indicators $\bar{\eta}_{H}(K)$.

Refine. Refine the marked elements (and some neighbors for mesh conformity) and update the mesh for the primal and the dual problem. Update the micro meshes of the sampling domains corresponding to the refined macro elements (see below for a discussion about an optimal coupling).

Remark 9. If a macro element is not marked for refinement, we carry over the solution of the corresponding microproblems from the current iteration to the next. This allows to significantly reduce the number of microproblems that need to be computed. We refer to [37, Section 4.1] for details.

In the residual-based adaptive FE-HMM case, the optimal coupling between the macro and micro mesh sizes for the data approximation error of the estimator is given - under appropriate assumptions - by $h_{K} \propto \varepsilon\left(H_{K}\right)^{\frac{p}{2 q}}$. Here $p$ and $q$ refer to the polynomial degree of the (primal) macro and micro FE spaces, respectively [37. Such a priori coupling conditions can be derived if we fix the way we measure the error (e.g., the energy norm for the residual-based adaptive FE-HMM). For error in quantities of interest, such coupling will depend on $J(\cdot)$, that is arbitrary (except for being linear and bounded). It would therefore be beneficial to introduce an a posteriori error estimator on the micro problems and couple the macro and micro refinement based on the experimental convergence rate of these error estimators.

As an alternative, a reduced basis technique could be used. There, a few representative basis functions with a large information content are computed once at an offline stage using a highly refined micro mesh. Typically, only a few reduced basis functions are needed if there is appropriate regularity with respect to the slow parameter. In the online stage, the reduced basis functions are used to approximate the solution of the micro problems with high accuracy, which renders an adaptive refinement of the micro meshes needless. We refer the reader to [51, where reduced basis combined with DWR-FE-HMM is studied. 


\section{Proofs of the main results}

In this section we give the proof of our main results described in Section 3.3 .

Proof of Theorem 5 (Exact DWR FE-HMM error representation). We proceed in two steps. First, we use duality arguments to state the error in a quantity of interest that still depends on the unknown dual function $z^{0}$. Second, we replace all numerically unavailable quantities, such as $z^{0}, a^{0}$ or $f$, by their computable, discrete approximations.

Step I. We want to find the exact error representation for the error $e^{H}:=u^{0}-u^{H M M}$ in the quantity of interest $J(\cdot)$, i.e.,

The dual problem 23

$$
J\left(e^{H}\right)=J\left(u^{0}\right)-J\left(u^{H M M}\right) .
$$

$$
B_{0}\left(\varphi, z^{0}\right)=J(\varphi)
$$

holds for all $\varphi \in H_{0}^{1}(\Omega)$. We therefore choose $\varphi=e^{H}$ to obtain

$$
J\left(e^{H}\right)=B_{0}\left(e^{H}, z^{0}\right) .
$$

We next make the estimate computable. In (46), the dual solution $z^{0}$ is the exact solution of (23). Furthermore, the bilinear form $B_{0}(\cdot, \cdot)$ involves the exact homogenized tensor, and the primal solution $u^{0}$ of (21) depends on the exact right hand side $f$. As these quantities can in general not be evaluated exactly in the a posteriori error estimate, we replace them by computable quantities as follows. We start by replacing the exact dual solution $z^{0}$ by the computable FE-HMM dual solution $z^{\mathscr{H} \mathscr{M} \mathscr{M}}$ in $(21)$. We obtain

$$
J\left(u^{0}-u^{H M M}\right)=B_{0}\left(u^{0}-u^{H M M}, z^{\mathcal{H M M}}\right)+B_{0}\left(u^{0}-u^{H M M}, z^{0}-z^{\mathcal{H} \mathfrak{M M}}\right) .
$$

The second and third terms of the right-hand side of (47) will be a contribution to the data approximation error. The first term of the right-hand side of (47) is further split into three parts,

$$
B_{0}\left(u^{0}-u^{H M M}, z^{\mathcal{H} \mathfrak{M M}}\right)=\int_{\Omega} f^{H} z^{\mathcal{H M M}} d x-\int_{\Omega}\left(f^{H}-f\right) z^{\mathcal{H} \mathfrak{M M}} d x-B_{0}\left(u^{H M M}, z^{\mathcal{H O M M}}\right),
$$

where $f^{H}$ is a a computable approximation of $f$ the right-hand side of problem (3).

Step II. We then substitute the flux $a^{0} \nabla u^{H M M}(x)$ involving the exact homogenized tensor $a^{0}(x)$ by the computable multiscale flux (34) in the third term of the right-hand of (48) and obtain

$$
\begin{aligned}
B_{0}\left(u^{H M M}, z^{\mathcal{H} \mathfrak{M M}}\right) & =\sum_{K \in \mathcal{T}_{H}} \int_{K}\left(a^{0}(x) \nabla u^{H M M}\right) \cdot \nabla z^{\mathcal{H} \mathfrak{M M}} d x=\sum_{K \in \mathcal{T}_{H}} \int_{K}\left(\Pi_{a^{\varepsilon} \nabla u_{K}^{h}}(x)\right) \cdot \nabla z^{\mathcal{H} \mathfrak{M} \mathcal{M}} d x \\
& -\sum_{K \in \mathcal{T}_{H}} \int_{K}\left(\Pi_{a^{\varepsilon} \nabla u_{K}^{h}}(x)-a^{0}(x) \nabla u^{H M M}\right) \cdot \nabla z^{\mathcal{H} \mathfrak{M M}} d x .
\end{aligned}
$$

Finally, by integrating 49 by part we can separate the estimate into interior element terms and jump terms

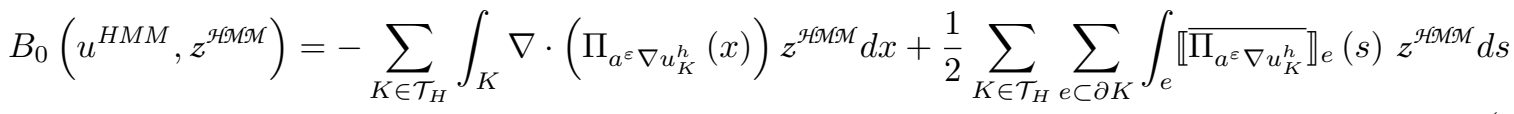

$$
\begin{aligned}
& -\sum_{K \in \mathcal{T}_{H}} \int_{K}\left(\Pi_{a^{\varepsilon} \nabla u_{K}^{h}}(x)-a^{0}(x) \nabla u^{H M M}\right) \cdot \nabla z^{\mathcal{H} \mathfrak{M M}} d x
\end{aligned}
$$

where we used the definition of the jump of multiscale fluxes $(36)$. Finally, we plug $(48)$ and (50) into (47) and obtain the error representation formula

$$
J\left(u^{0}-u^{H M M}\right)=\sum_{K \in \mathcal{T}_{H}} \eta_{H}(K)+\xi_{H}(K),
$$

where $\eta_{H}(K)$ and $\xi_{H}(K)$ are defined in $(38)$ and $(39)$, respectively, and the proof is complete.

Up to this point we did not assume specific regularity on the oscillating tensor, $a^{\varepsilon}$ or make any spatial assumptions such as periodicity in the micro scale, to derive our error estimates. Furthermore, we 
kept both sampling domain size and boundary conditions of the micro problem for the FE-HMM rather general. We now find estimates of the data approximation error (39). Notice first that (see (35))

$$
\left\|a^{0}(x) \nabla v^{H}(x)-\Pi_{a^{\varepsilon} \nabla v_{K}^{h}}(x)\right\|_{L^{2}(K)}=\left\|a^{0}(x) \nabla v^{H}(x)-\Pi_{a_{K}^{0} \nabla v^{H}}(x)\right\|_{L^{2}(K)} .
$$

Recalling the tensors $a^{0}, \bar{a}_{K}^{0}$ introduced in Section 2.1, we then consider the decomposition

$$
\begin{aligned}
& \left\|a^{0}(x) \nabla v^{H}(x)-\Pi_{a_{K}^{0} \nabla v^{H}}(x)\right\|_{L^{2}(K)} \leq \underbrace{\left\|a^{0}(x) \nabla v^{H}(x)-\Pi_{a^{0} \nabla v_{K}^{H}}(x)\right\|_{L^{2}(K)}}_{e_{I}} \\
& +\underbrace{\left\|\Pi_{a^{0} \nabla v_{K}^{H}}(x)-\Pi_{\bar{a}_{K}^{0} \nabla v_{K}^{H}}(x)\right\|_{L^{2}(K)}}_{e_{I I}}+\underbrace{\left\|\Pi_{\bar{a}_{K}^{0} \nabla v_{K}^{H}}(x)-\Pi_{a_{K}^{0} \nabla v_{K}^{H}}(x)\right\|_{L^{2}(K)}}_{e_{I I I}},
\end{aligned}
$$

where $\Pi_{a^{0} \nabla v_{K}^{H}}(x)$ and $\Pi_{\bar{a}_{K}^{0} \nabla v_{K}^{H}}(x)$ are the interpolating polynomials based on the quadrature nodes $x_{K_{\ell}} \in K$ with function values given by $a^{0}\left(x_{K_{\ell}}\right) \nabla v^{H}\left(x_{K_{\ell}}\right)$ and $\bar{a}_{K}^{0}\left(x_{K_{\ell}}\right) \nabla v^{H}\left(x_{K_{\ell}}\right)$, respectively. The task is now to estimate $e_{I}, e_{I I}, e_{I I I}$.

Lemma 10. Assume that $(\boldsymbol{Q})$ and $(\mathbf{H 2})$ hold and that $\left.a(\cdot, y)\right|_{K}$, is constant for any $K \in \mathcal{T}_{H}$. Then

$$
e_{I}=0
$$

Proof. Under the assumptions (H2) and $\left.a(\cdot, y)\right|_{K}$, constant, the homogenized tensor $a^{0}(x)$ is constant in each $K \in \mathcal{T}_{H}$ and the interpolation 34 is exact, i.e. $\Pi_{a^{0} \nabla v_{K}^{H}}(x) \equiv a^{0}(x) \nabla v^{H}(x)$ for $x \in K$ and $v^{H} \in V^{p}\left(\Omega, \mathcal{T}_{H}\right)$.

We notice that for piecewise linear elements, defining $\Pi_{a^{0} \nabla v_{K}^{H}}(x)=a^{0}\left(x_{K}\right) \nabla v_{K}^{H}$, where $x_{K} \in K$, we obtain $e_{I} \leq C H$ (using appropriate smoothness of $a^{0}(x)$ ). Using this estimate in the proof of Theorem 7 (see below) proves Remark 43

To estimate $e_{I I}$ and $e_{I I I}$ we need to relate the error of two interpolating polynomials to the error of the function values at the corresponding interpolation nodes. This relation will allow us to find similar estimates of the data approximation error as obtained in [37.

Lemma 11. For $v, \tilde{v} \in C^{0}(K)$ we consider the interpolation polynomials $\Pi_{v}(x) \in \mathcal{P}^{p-1}(K)$ and $\Pi_{\tilde{v}}(x) \in$ $\mathcal{P}^{p-1}(K)$, respectively, defined by $(32)$ based on the nodes of a $Q F\left\{x_{K_{\ell}}, \omega_{K_{\ell}}\right\}_{\ell=1}^{\mathcal{L}}$ that satisfies (Q) and (31). Then

$$
\left\|\Pi_{v}(x)-\Pi_{\tilde{v}}(x)\right\|_{L^{2}(K)} \leq C \sqrt{|K|} \max _{1 \leq \ell \leq \mathcal{L}}\left|v_{\ell}-\tilde{v}_{\ell}\right|,
$$

where $|K|$ denotes the measure of the macro element $K$, the constant $C$ only depends on the dimensionality $d$, the polynomial degree $p$, the shape regularity constant $\gamma$ and the quadrature formula.

Proof. Using (32) we can introduce an interpolating polynomial $\Pi_{v-\tilde{v}}(x) \in \mathcal{P}^{p-1}(K)$ s.t. $\Pi_{v-\tilde{v}}\left(x_{K_{\ell}}\right)=$ $v\left(x_{K_{\ell}}\right)-\tilde{v}\left(x_{K_{\ell}}\right), \ell=1, \ldots, \mathcal{L}$. We use the $C^{1}-$ diffeomorphism $F_{K}$ between $K$ and the reference element $\hat{K}$ and define $\widehat{\Pi_{v-\tilde{v}}}(\hat{x}):=\Pi_{v-\tilde{v}}\left(F_{K}(\hat{x})\right)=\Pi_{v-\tilde{v}}(x)$. It is well-known that (see for example [21])

$$
\left\|\Pi_{v-\tilde{v}}(x)\right\|_{L^{2}(K)} \leq C\left|\operatorname{det}\left(\partial F_{K}\right)\right|^{1 / 2}\left\|\widehat{\Pi_{v-\tilde{v}}}(\hat{x})\right\|_{L^{2}(\hat{K})} .
$$

Given a basis $\left\{\hat{q}_{j}\right\}_{j=1}^{\mathcal{L}}$ of $\mathcal{P}^{p-1}(\hat{K})$ we expand $\widehat{\Pi_{v-\tilde{v}}}(\hat{x})=\sum_{j=1}^{\mathcal{L}} \hat{\beta}_{j} \hat{q}_{j}(\hat{x})$, with coefficients $\hat{\beta}_{j} \in \mathbb{R}$, $j=1, \ldots, \mathcal{L}$. Evaluating the above formula at $\hat{x}_{\ell}=F_{K}^{-1}\left(x_{K_{\ell}}\right)$ we obtain $\widehat{\Pi_{v-\tilde{v}}}\left(\hat{x}_{\ell}\right)=\sum_{j=1}^{\mathcal{L}} \hat{A}_{\ell j} \hat{\beta}_{j}$, where $\hat{A}_{\ell j}=\hat{q}_{j}\left(\hat{x}_{\ell}\right)$. This last relation read in vector form $\hat{\boldsymbol{A}} \hat{\boldsymbol{\beta}}=\delta \boldsymbol{v}$, where $(\hat{\boldsymbol{A}})_{\ell j}=\hat{A}_{\ell j},(\hat{\boldsymbol{\beta}})_{j}=\hat{\boldsymbol{\beta}}_{j}$ and $(\delta \boldsymbol{v})_{\ell}=v\left(x_{K_{\ell}}\right)-\tilde{v}\left(x_{K_{\ell}}\right)$. From Lemma 3 it follows that $\hat{\boldsymbol{A}}$ is invertible. Then we can estimate (51) 
further as follows

$$
\begin{aligned}
\left\|\Pi_{v-\tilde{v}}(x)\right\|_{L^{2}(K)} & \leq C\left|\operatorname{det}\left(\partial F_{K}\right)\right|^{1 / 2}\left\|\widehat{\Pi_{v-\tilde{v}}}(\hat{x})\right\|_{L^{2}(\hat{K})} \\
& \leq C \sqrt{|K|}\left\|\sum_{\ell=1}^{\mathcal{L}} \hat{\beta}_{\ell} \hat{q}_{\ell}(\hat{x})\right\|_{L^{2}(\hat{K})} \\
& \leq C \sqrt{|K|} \sum_{\ell=1}^{\mathcal{L}}\left\|\hat{q}_{\ell}(\hat{x})\right\|_{L^{2}(\hat{K})}\|\hat{\boldsymbol{\beta}}\|_{\infty} \\
& \leq C \sqrt{|K|}\left\|\hat{\boldsymbol{A}}^{-1}(\delta \boldsymbol{v})\right\|_{\infty} \leq C \sqrt{|K|} \max _{1 \leq \ell \leq \mathcal{L}}\left|v\left(x_{K_{\ell}}\right)-\tilde{v}\left(x_{K_{\ell}}\right)\right|,
\end{aligned}
$$

where $\|\cdot\|_{\infty}$ is the supremum matrix and vector norm, respectively, and the constant $C$ only depends on the dimensionality $d$, the polynomial degree $p$, the shape regularity constant $\gamma$, the quadrature formula $\left\{\hat{x}_{\ell}, \hat{\omega}_{\ell}\right\}_{\ell=1}^{\mathcal{L}}$ and the basis $\left\{\hat{q}_{j}\right\}_{j=1}^{\mathcal{L}}$ of $\mathcal{P}_{d}^{p-1}(\hat{K})$.

We can next relate the modeling and the micro errors to the various numerically homogenized tensors.

Lemma 12. Assume that (Q) holds, then we have

$$
e_{I I} \leq C \max _{1 \leq \ell \leq \mathcal{L}}\left\|a^{0}\left(x_{K_{\ell}}\right)-\bar{a}_{K}^{0}\left(x_{K_{\ell}}\right)\right\|_{F}\left\|\nabla v^{H}(x)\right\|_{L^{2}(K)}
$$

and

$$
e_{I I I} \leq C \max _{1 \leq \ell \leq \mathcal{L}}\left\|\bar{a}_{K}^{0}\left(x_{K_{\ell}}\right)-a_{K}^{0}\left(x_{K_{\ell}}\right)\right\|_{F}\left\|\nabla v^{H}(x)\right\|_{L^{2}(K)},
$$

where the constant $C$ only depends on the dimensionality d, the polynomial degree $p$ of the FEM for $u^{H}$, the shape regularity constant and the quadrature formula.

Proof. Both estimates use Lemma 11. For example for $e_{I I}$ we have

$$
\begin{aligned}
e_{I I} & =\left\|\Pi_{a^{0} \nabla v_{K}^{H}}(x)-\Pi_{\bar{a}_{K}^{0} \nabla v_{K}^{H}}(x)\right\|_{L^{2}(K)} \\
& \leq C \sqrt{|K|} \max _{1 \leq \ell \leq \mathcal{L}}\left\|\left(a^{0}\left(x_{K}\right)-\bar{a}_{K}^{0}\left(x_{K_{\ell}}\right)\right) \nabla v^{H}\left(x_{K_{\ell}}\right)\right\|_{\infty} \\
& \leq C\left(\max _{1 \leq \ell \leq \mathcal{L}}\left\|\left(a^{0}\left(x_{K_{\ell}}\right)-\bar{a}_{K}^{0}\left(x_{K_{\ell}}\right)\right)\right\|_{F}\right) \sum_{\ell=1}^{\mathcal{L}} \sqrt{|K|}\left(\sum_{j} \partial_{j} v^{H}\left(x_{K_{\ell}}\right)^{2}\right)^{1 / 2} \\
& \leq C\left(\max _{1 \leq \ell \leq \mathcal{L}}\left\|a^{0}\left(x_{K_{\ell}}\right)-\bar{a}_{K}^{0}\left(x_{K_{\ell}}\right)\right\|_{F}\right)\left\|\nabla v^{H}(x)\right\|_{L^{2}(K)},
\end{aligned}
$$

where we used that

$$
\begin{aligned}
\sum_{\ell=1}^{\mathcal{L}} \sqrt{|K|}\left(\sum_{j} \partial_{j} v^{H}\left(x_{K_{\ell}}\right)^{2}\right)^{1 / 2} & \leq C\left(\sum_{\ell=1}^{\mathcal{L}} \omega_{K_{\ell}} \sum_{j} \partial_{j} v^{H}\left(x_{K_{\ell}}\right)^{2}\right)^{1 / 2} \\
& \leq C\left\|\nabla v^{H}(x)\right\|_{L^{2}(K)} .
\end{aligned}
$$

This last inequalities are obtained using the assumption $(\mathbf{Q})$, a scaling argument as well as the equivalence of norms on a finite dimensional space (see for example [52, Lemma 5]). The proof of (53) is obtained similarly.

Proof of Theorem 7 (Error bound for data approximation error). Summing over the local data 
approximation error defined in (39) gives

$$
\begin{aligned}
\sum_{K \in \mathcal{T}_{H}} \xi_{H}(K)= & \underbrace{\sum_{K \in \mathcal{T}_{H}} \int_{K}\left[\Pi_{a^{\varepsilon} \nabla u_{K}^{h}}(x)-a^{0}(x) \nabla u^{H M M}\right] \cdot \nabla z^{\mathfrak{H} \mathfrak{M M}} d x}_{I} \\
& +\underbrace{\sum_{K \in \mathcal{T}_{H}} B_{0, K}\left(u^{0}-u^{H M M}, z^{0}-z^{\mathcal{H} \mathfrak{M M}}\right)}_{I I} \\
& +\underbrace{\sum_{K \in \mathcal{T}_{H}} \int_{K}\left(f-f^{H}\right) z^{\mathfrak{H} \mathcal{M M}} d x}_{I I I} .
\end{aligned}
$$

In view of the definitions 16 and 17 for $r_{M I C}$ and $r_{M O D}$, respectively, we have

$$
|I| \leq C\left(r_{M I C}+r_{M O D}\right)
$$

where we used Lemmas 10 and 12 and the Cauchy-Schwarz inequality. We note that both $\left\|\nabla u^{H}\right\|_{L^{2}(\Omega)}$ and $\left\|\nabla z^{\mathfrak{H} \mathfrak{M} \mathscr{M}}\right\|_{L^{2}(\Omega)}$ can be bounded by $\tilde{C}\|f\|_{L^{2}(\Omega)} \leq C$. Using Cauchy-Schwarz inequality we obtain

$$
\begin{aligned}
|I I| & \leq C\left\|\nabla u^{0}-\nabla u^{H M M}\right\|_{L^{2}(\Omega)}\left\|\nabla z^{0}-\nabla z^{\mathcal{H} \mathfrak{H M M}}\right\|_{L^{2}(\Omega)} \\
& \leq C\left(H^{p}+r_{M I C}+r_{M O D}\right)\left(H^{\tilde{p}}+r_{\mathcal{M I C}}+r_{\mathcal{M O D}}\right) .
\end{aligned}
$$

and similarly

$$
|I I I| \leq C\left\|f-f^{H}\right\|_{L^{2}(\Omega)}
$$

By noting that both $r_{M I C}$ and $r_{M I C}$ can be estimated by $C\left(\frac{h}{\varepsilon}\right)^{2 q}$ under assumption (H1) and that both $r_{M O D}$ and $r_{\mathcal{M O D}}$ are equal to zero if $S^{q}\left(K_{\delta_{\ell}}, \mathcal{T}_{h}\right) \subset W_{\text {per }}^{1}\left(K_{\delta_{\ell}}\right)$ and $\frac{\delta}{\varepsilon} \in \mathbb{N}$ or can be estimated by $C\left(\varepsilon+\left(\frac{\varepsilon}{\delta}\right)\right)$ if $S^{q}\left(K_{\delta_{\ell}}, \mathcal{T}_{h}\right) \subset H_{0}^{1}\left(K_{\delta_{\ell}}\right)(\delta>\varepsilon)$ we conclude the proof.

Proof of Corollary 7 (Consistency with single scale DWR). Under assumption (H2) and if $\left.a(\cdot, y)\right|_{K}$, is constant for any $K \in \mathcal{T}_{H}$, assuming further exact micro functions, we have $a^{0}=\bar{a}_{K}^{0}=a_{K}^{0}$ in each $K \in \mathcal{T}_{H}$. It then follows that $a^{0}(x) \nabla v^{H}(x)=\Pi_{a^{\varepsilon} \nabla v_{K}^{h}}(x)$ and thus 26) and (38) are equal. Next for exact micro functions the term $C\left(\frac{h}{\varepsilon}\right)^{2 q}$ vanishes in the data approximation error and finally if $\frac{\delta}{\varepsilon} \in \mathbb{N}$ $r_{M O D}=0$ and the proof of the corollary is complete.

\section{$5 \quad$ Numerical experiments}

In this section we will show numerical experiments that confirm the efficiency of the goal-oriented, adaptive FE-HMM and the effectivity of the estimated error in a quantity of interest. Our numerical experiments were carried out using an implementation in Matlab; this implementation is based on the FE-HMM code presented in in [53. The code for the mesh bisection is based in part on $i$ FEM (see [54]) and the code for higher-order FEM is based in part on [55. We will also compare our goal oriented adaptive strategy with the residual based adaptivity in the energy norm developed earlier for the FE-HMM [36, 37]. There we have proved a posteriori upper and lower bounds for the error between the macroscopic FE-HMM solution $u^{H M M}$ and the homogenized solution $u^{0}$ be the solution of the problem (3). These estimates read as follow

$$
\begin{gathered}
\left\|u^{0}-u^{H M M}\right\|_{H^{1}(\Omega)}^{2} \leq C\left(\eta_{H, \text { Residual }}(\Omega)^{2}+\xi_{H}(\Omega)^{2}\right) . \\
\eta_{H, \text { Residual }}(K)^{2} \leq C\left(\left\|u^{0}-u^{H M M}\right\|_{H^{1}\left(\omega_{K}\right)}^{2}+\xi_{H}\left(\Omega_{K}\right)^{2}\right),
\end{gathered}
$$

where the domain $\Omega_{K}$ consists of all elements sharing at least one side with $K$. We refer to [36, 37] for a precise definition of $\eta_{H, \text { Residual }}(\Omega)$ and $\xi_{H, \text { Residual }}(\Omega)$. We emphasize that $\eta_{H, \text { Residual }}(\Omega)$ is different from $\eta_{H}(\Omega)$ defined in $(38)$. We consider a problem (which is chosen similarly to the one-dimensional 
problems presented in [22]) where we are interested in the pointwise error and the pointwise directional derivative error at a certain point $x^{\star}$. We consider the equation

$$
\begin{aligned}
-\nabla \cdot\left(a^{\varepsilon}(x) \nabla u^{\varepsilon}\right) & =f & & \text { in } \Omega, \\
u^{\varepsilon} & =g_{D} & & \text { on } \Gamma_{D}=\partial \Omega,
\end{aligned}
$$

on a domain $\bar{\Omega}=(0,1)^{2}$. We choose $\varepsilon=10^{-5}$ and set the tensor to

$$
a\left(x, \frac{x}{\varepsilon}\right):=\tilde{a}^{\varepsilon}\left(\frac{x}{\varepsilon}\right) a^{0}(x),
$$

where the exact homogenized tensor $a^{0}(x)$ is given as

$$
a^{0}(x):=\left(9 e^{-1000\left(x_{1}-0.5\right)^{2}-1000\left(x_{2}-0.5\right)^{2}}+1\right) I_{2} .
$$

Here

$$
\tilde{a}^{\varepsilon}(x):=\frac{16}{15}\left(\sin \left(2 \pi \frac{x_{1}}{\varepsilon}\right)+\frac{5}{4}\right)\left(\cos \left(2 \pi \frac{x_{2}}{\varepsilon}\right)+\frac{5}{4}\right),
$$

whose coefficients are chosen in such a way that they give the unit tensor when homogenized. We define the exact solution as

$$
u^{0}(x):=100\left(1-x_{1}\right)^{2} x_{1}\left(1-x_{2}\right)^{2} x_{2} e^{-20\left(x_{1}-\frac{1}{3}\right)^{2}-\left(x_{2}-\frac{1}{4}\right)^{2}},
$$

which determines the Dirichlet boundary conditions as $g_{D}=u^{0}$ on $\partial \Omega$, and choose the right hand side $f$ accordingly. An illustration of the exact solution and the homogenized tensor are given in Figure 1. We

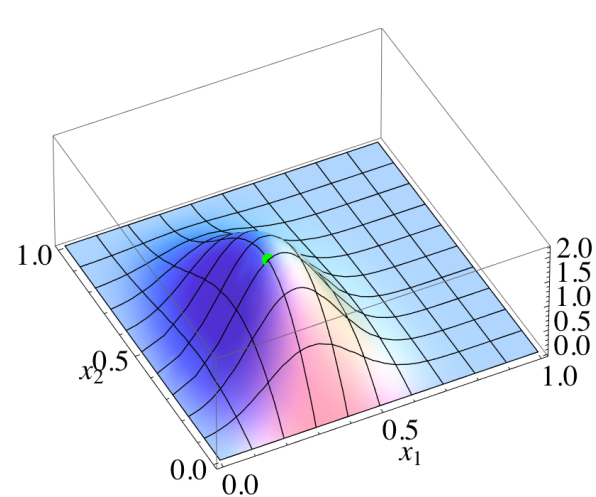

(a) Exact homogenized solution $u^{0}(x)$. We are interested in the pointwise error and the directional derivative error at the point $x^{\star}=(0.3,0.3)$, which is marked in green.

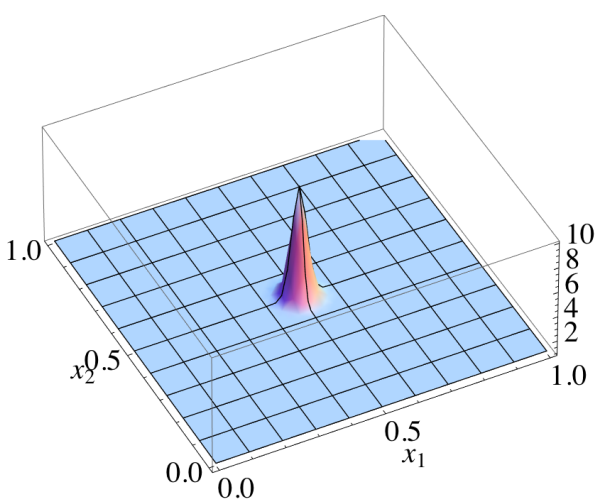

(b) Exact homogenized tensor $a^{0}(x)$.

Figure 1: Exact solution and tensor of the goal-oriented problems of Section 5 .

use piecewise linear macro FE for the solution $u^{H M M}$ of the primal problem and piecewise quadratic FE for the solution $z^{\mathcal{H M M}}$ of the macro dual problem. The micro problems are solved with piecewise linear FE.

As a measure of the quality of our error estimator, we define the effectivity index

$$
\text { Eff }:=\left|\frac{\eta_{H}(\Omega)}{J\left(u^{0}-u^{H M M}\right)}\right|,
$$

which ideally should be equal to one. We denote by $\hat{h}:=\left(N_{m i c}\right)^{-1 / d}$ the scaled (i.e. independent of $\varepsilon$ ) micro mesh size, where $N_{\text {mic }}$ refers to the degrees of freedom of the micro problems in one micro sampling domain corresponding to a certain macro element.

For the macro mesh we start for both problems from a mesh with 441 DOF. We do not choose a uniform grid but one where the points are slightly distorted compared to uniform mesh, see Figure 7. 
The distortion will resemble to more realistic meshes originating from practical applications and reduce effects of cancelation. We use an initial mesh size for the micro mesh of $\hat{h}=\frac{1}{8}$ and $\delta=\varepsilon=10^{-5}$ with periodic boundary conditions for the micro problems.

For the marking strategy we will follow the maximum marking strategy [22] for the quantity of interest $J\left(u^{0}-u^{H M M}\right)$. An element $K$ of the mesh is refined if

$$
\frac{\tilde{\eta}_{H}(K)}{\max _{\tilde{K} \in \mathcal{T}_{H}} \tilde{\eta}_{H}(\tilde{K})} \geq \vartheta
$$

where $0<\vartheta<1$ is a user-defined parameter. We choose $\vartheta=0.25$. The mesh is refined using the newest vertex bisection in its implementation of $i$ FEM [54.

We choose a coupling of $\hat{h} \propto H_{k}$ for both the solution of the primal and dual problem and for the evaluation of the a posteriori estimator. For numerical experiments with different coupling schemes, we refer to [4]. We recall that for macro elements that were not refined, the solution of the microproblems from the previous iteration should be re-used (see Remark 9.

\subsection{Pointwise error}

In our first experiment, we choose the quantity of interest to be the pointwise error at the point $x^{\star}=$ $(0.3,0.3)$; the quantity of interest is

$$
J(u):=u\left(x^{\star}\right) .
$$

The point $x^{\star}$ coincides with a node of the mesh and the exact quantity of interest is given by $J(u) \approx$ 2.10813. In Figure 2 we compare the error for the uniform refinement, the global residual-based refinement and the goal-oriented refinement of the FE-HMM. The error of all three methods shows an asymptotic convergence rate of $\mathcal{O}\left(N_{m a c}^{-1}\right)$. The pointwise error is smaller for the uniform or residual-based adaptivity

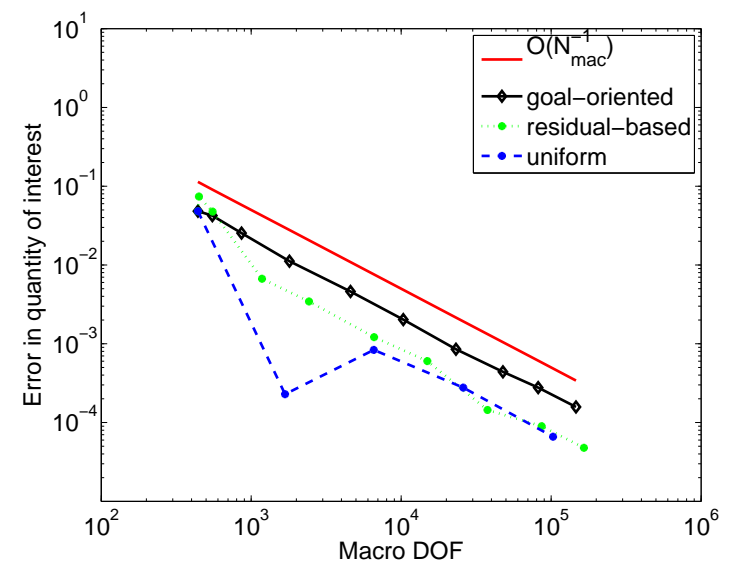

(a) Error for uniform, global residual-based and goaloriented refinement.

Figure 2: Errors for the goal-oriented problem described in Section 5.1 .

than it is for the goal-oriented adaptivity. But the DWR FE-HMM also provides an estimation of the actual error in the quantitity of interest, which serves as a stopping criterion. In Figure 3 we present the effectivity index $\frac{\eta_{H, \text { Residual }}(\Omega)}{\left|J\left(e^{H}\right)\right|}$ and $\frac{\eta_{H}(\Omega)}{\left|J\left(e^{H}\right)\right|}$ for the residual-based and the goal-oriented adaptivity, respectively. While the effectivity index for goal-oriented adaptivity is close to one, the residual-based estimate approximates the error in the energy norm and therefore gives an effectivity index which is not suitable as a stopping criterion; the residual-based error estimate further depends on an unspecific constant. In the first two iterations for the DWR FE-HMM, the error is underestimated, leading to an effectivity index smaller than one. Additional a posteriori error estimates on the different components of the data approximation error can be used to avoid the underestimation of the error, we refer to [28, 27] and to the comment at the end of Section 3.3 . 
Finally, we compare in Figure 4 the mesh of the goal-oriented and the residual-based adaptivity after 4 iterations. We see that the mesh is highly refined around $x^{\star}$ and the "peak" of the tensor for the goaloriented adaptivity, whereas the residual-based adaptivity obviously does not especially refine around $x^{\star}$.

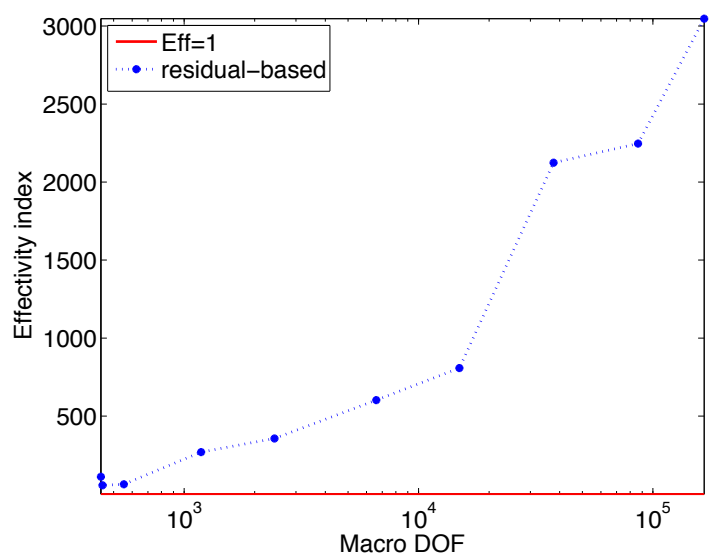

(a) Effectivity index for the global residual-based adaptivity.

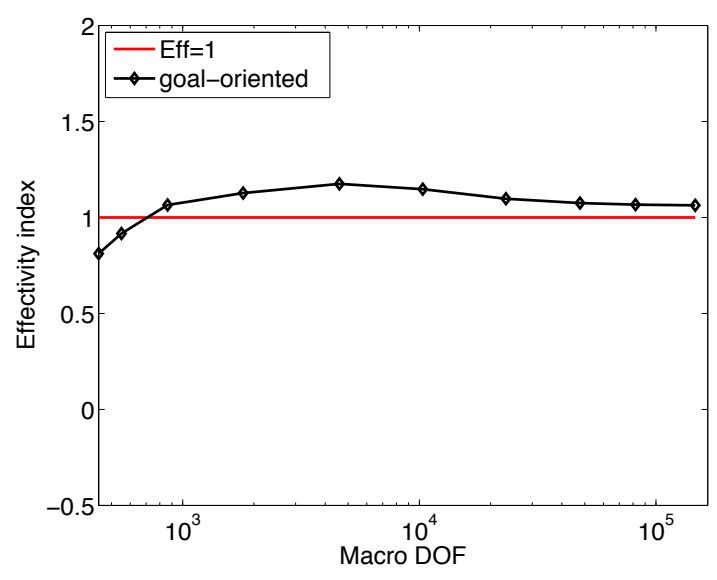

(b) Effectivity index for the goal-oriented adaptivity.

Figure 3: Effectivity index (residual based and goal-oriented adaptivity) for the problem described in Section 5.1

\subsection{Pointwise directional derivative error}

We choose our quantity of interest to be the directional derivative error at the point $x^{\star}=(0.3,0.3)$ (slightly off the peak of the "bump") in direction $n=(1 / \sqrt{2}, 1 / \sqrt{2})$, such that

$$
J(u):=\nabla u\left(x^{\star}\right) \cdot n
$$

The exact solution is given by $J \approx 3.25819$. As the pointwise derivatives of $\mathrm{FE}$ solutions are in general not defined on edges of elements, we approximate the functional $(60)$ at point $x^{\star} \in \Omega$ with a regularized output functional $J(u)=\frac{1}{\left.S^{\epsilon}\right]} \int_{S^{\epsilon}} \nabla u \cdot n d x$, where the small domain $S^{\epsilon}$ is an $\epsilon$-ball centered around the point $x^{\star}$, see [25]. In Figure 5(a) we compare the error when using a uniform, a global residual-based (as in [37) and the DWR FE-HMM goal-oriented approach. We see that the pointwise derivative error $J\left(e^{H}\right)$ converges with an order of $\mathcal{O}\left(N_{m a c}^{-1 / 2}\right)$ for the uniform refinement and for the adaptive, residualbased refinement scheme. For the DWR FE-HMM we obtain a (mean) convergence rate of approximately $\mathcal{O}\left(N_{m a c}^{-3 / 2}\right)$, which varies between $\mathcal{O}\left(N_{m a c}^{-1}\right)$ and $\mathcal{O}\left(N_{m a c}^{-2}\right)$. We notice that the convergence rate is much faster than the $\mathcal{O}\left(N_{m a c}^{-1 / 2}\right)$ we would have in the global energy-norm. In Figure 5 (b) we see that the effectivity index for the DWR FE-HMM varies between 0.5 and 8 . The residual-based estimate in the energy norm involves an unspecific constant, varies between 7 and 120 and is not suitable for estimating the error in the quantity of interest. Therefore the residual-based estimate is also unsuitable as a reliable stopping criterion. The jagged line for the error and efficiency of the DWR FE-HMM is caused by the cancelation of errors; the jaggedness can also be seen in the single-scale DWR FEM case.

We illustrate in Figure 6 how the dual-weighted residual error estimates can be used to evaluate the quality of the numerical solution. As the exact error representation does not involve an unspecific constant, we have the possibility to specify - solely based on the error estimate - a confidence interval where we expect the exact quantity of interest to be. If the data approximation error is much smaller than the error estimator $\eta_{H}(\Omega)$, we can set $J\left(u^{H M M}\right)-\left|\eta_{H}(\Omega)\right|$ and $J\left(u^{H M M}\right)+\left|\eta_{H}(\Omega)\right|$ to be our confidence interval. We see in Figure 6 that except for iteration 1, the exact solution lies within the confidence interval. 


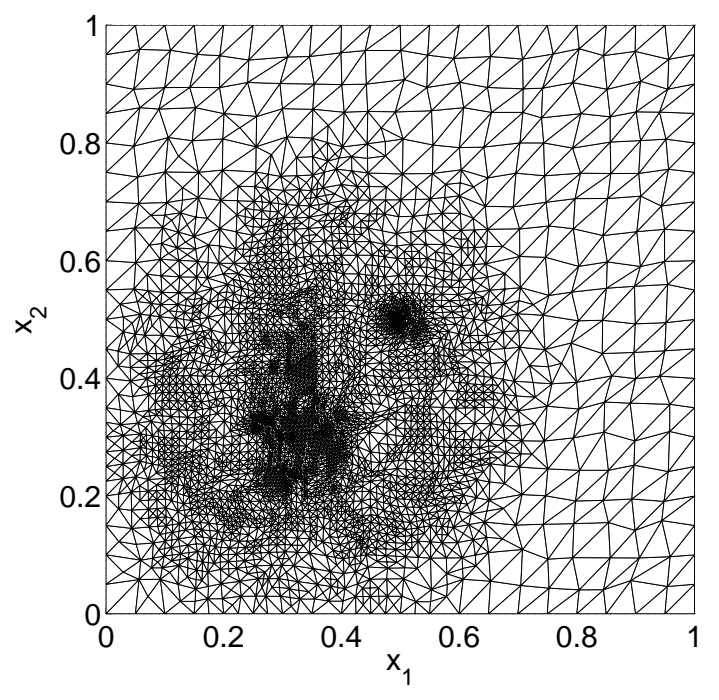

(a) Goal-oriented refinement $(4596$ DOF, $\left.J\left(u^{0}-u^{H M M}\right)=0.00461\right)$.

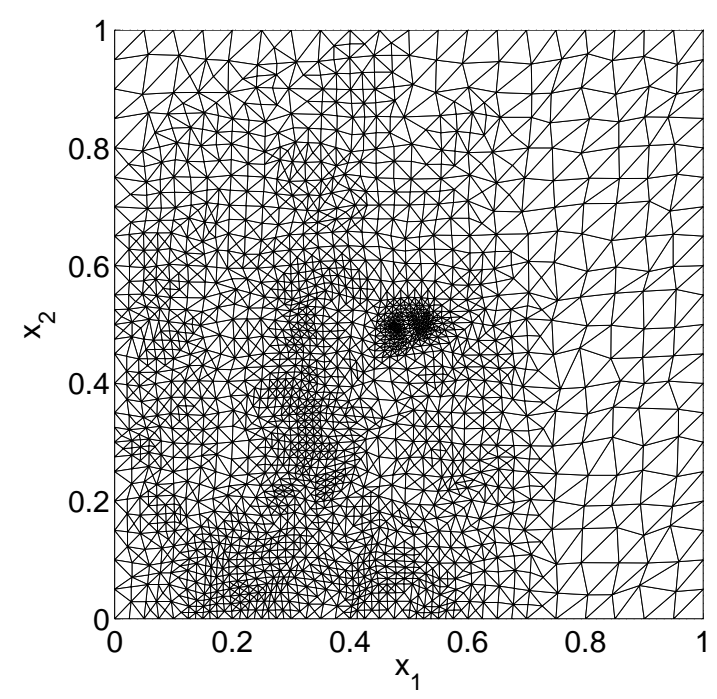

(b) Global residual-based refinement (2428 DOF, $\left.J\left(u^{0}-u^{H M M}\right)=0.00344\right)$.

Figure 4: Mesh after 4 iterations for global residual-based and goal-oriented refinement for the pointwise directional derivative problem.

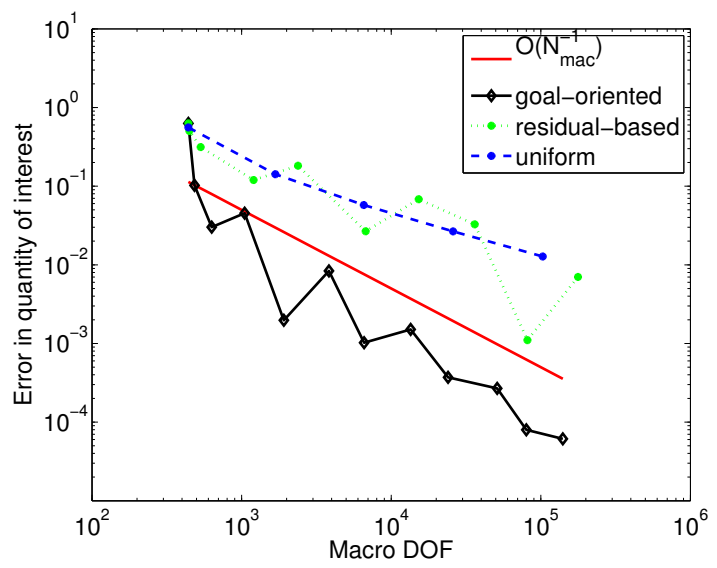

(a) Error for uniform, global residual-based and goaloriented refinement.

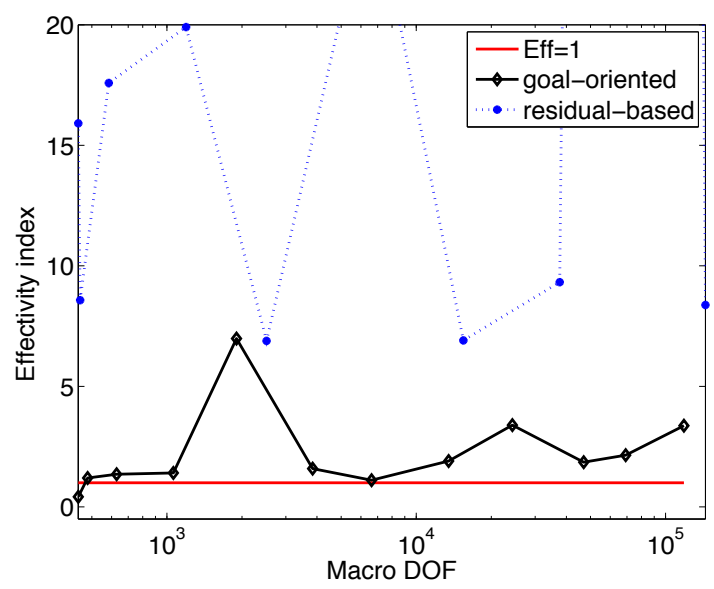

(b) Effectivity index for the global residual-based $\left(\frac{\eta_{H, \text { Residual }}(\Omega)}{\left|J\left(e^{H}\right)\right|}\right)$ and goal-oriented adaptivity.

Figure 5: Errors and effectivity index for the goal-oriented problem described in Section 5.2 .

Finally we show in Figure 7 the meshes after 4 iterations for both the goal-oriented and the residualbased adaptive FE-HMM. While the residual-based scheme mostly refines around the peak of the conductivity tensor, the goal-oriented additionally refines around the point of interest $x^{\star}$.

\section{Conclusion}

We have presented an a posteriori error analysis in quantities of interest for the FE-HMM and generalized the DWR approach for homogenization problems. An exact error representation has been derived and effective error indicators have been modeled based solely on micro computations on sampling domains within the physical computational domain. Rigorous upper bound on the data approximation errors have been established. These errors also take into account the variational crimes committed by our 


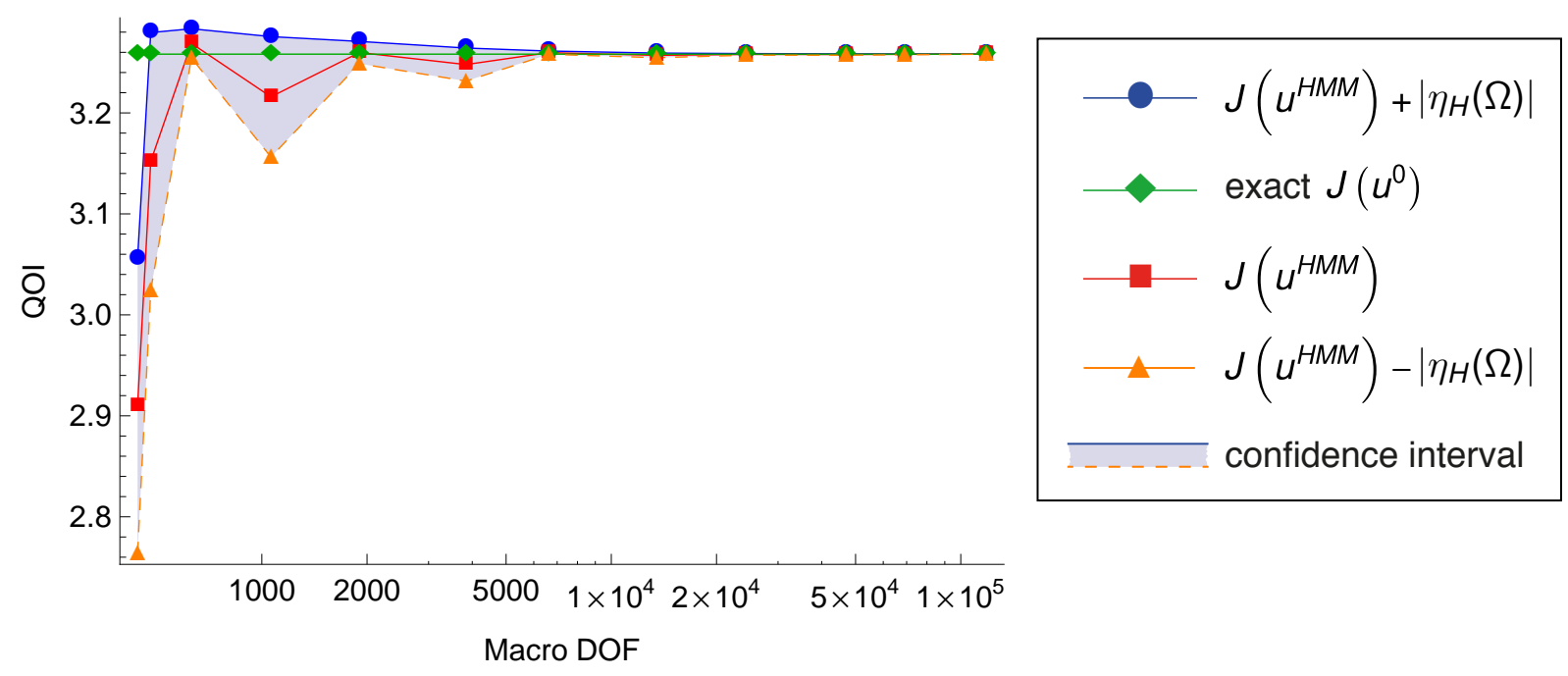

Figure 6: The use of duality-based goal-oriented FE-HMM allows to specify - solely based on the $a$ posteriori error estimate - a confidence interval (shown in shaded blue) where we expect the exact quantity of interest to be within. From iteration 2 on, the prediction is correct.

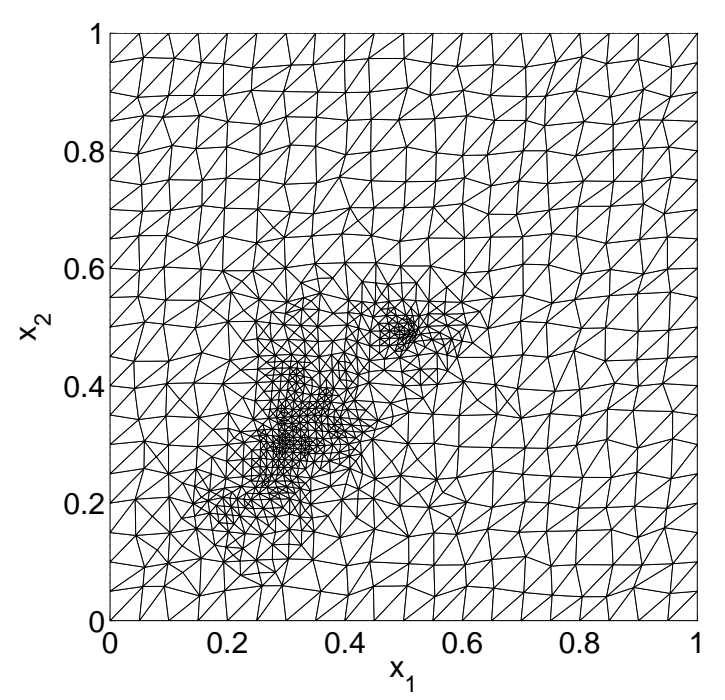

(a) Goal-oriented refinement (1041 DOF, $\left.J\left(u^{0}-u^{H M M}\right)=0.034\right)$

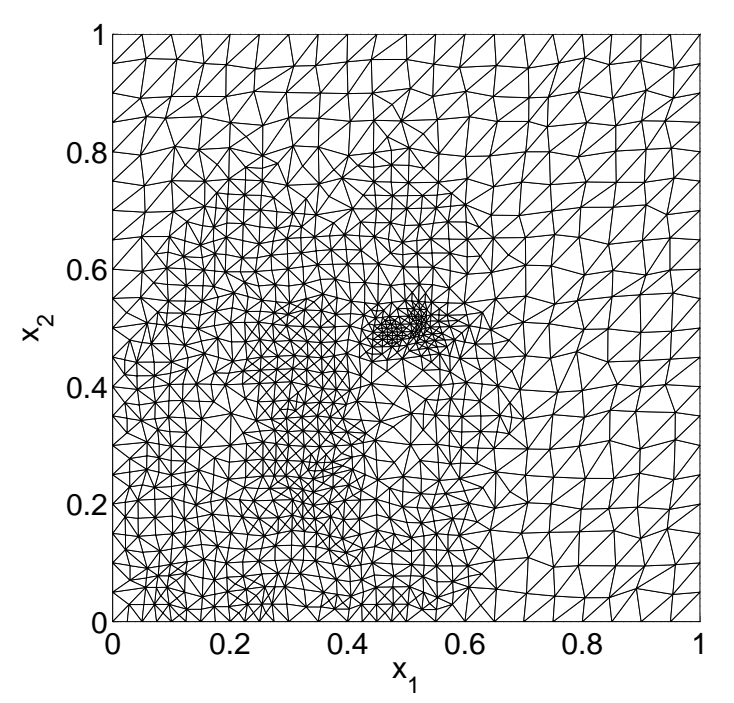

(b) Global residual-based refinement (1209 DOF, $\left.J\left(u^{0}-u^{H M M}\right)=0.113\right)$.

Figure 7: Mesh after 4 iterations for global residual-based and goal-oriented refinement for the pointwise directional derivative problem. 
computational strategy relying on numerical integration. Numerical experiments presented in the present paper confirm the efficiency of the proposed (multiscale) adaptive method in quantity of interests. We note that even for single scale problems, the DWR for FEM with numerical integration has not yet been analyzed. While we have provided an a priori upper bound for the data approximation error we did not provide an a posteriori error estimate for these quantities. This could be addressed by designing also an a posteriori error procedure for the micro solution in the sampling domains. For single scale problems, in the conforming case, a posteriori bounds for the data approximation errors were recently derived in [28]. For multiscale problems as considered here and with the proposed micro-to-macro approach, quantifying the modeling error and the error arising from variational crimes would also be required. This is a topic for future research. An alternative, to extend the framework proposed in this paper without using a posteriori error estimates for the micro solutions is to use reduced basis techniques (RB) for the micro solver. As shown in [56, a combination of the FE-HMM with RB permits to avoid simultaneous refinement as the $\mathrm{RB}$ space for the micro-solutions is computed very accurately in an offline stage independently of the macro mesh and then re-used in the macro adaptivity cycles.

\section{References}

[1] I. Babuška and J.E. Osborn. Generalized finite element methods: their performance and their relation to mixed methods. SIAM Journal on Numerical Analysis, 20(3):510-536, 1983.

[2] T.Y. Hou, X.H. Wu, and Z. Cai. Convergence of a Multiscale Finite Element Method for Elliptic Problems with Rapidly Oscillating Coefficients. Mathematics of Computation, 68(227):913-943, 1999.

[3] Y. Efendiev and T. Y. Hou. Multiscale Finite Element Methods: Theory and Applications. Springer, Berlin, 2009.

[4] A.M. Matache, I. Babuška, and Ch. Schwab. Generalized p-FEM in homogenization. Numerische Mathematik, 86(2):319-375, 2000.

[5] T.J.R. Hughes, G.R. Feijoo, L. Mazzei, and J.B. Quincy. The variational multiscale method-a paradigm for computational mechanics. Computer methods in applied mechanics and engineering, 166(1-2):3-24, 1998.

[6] V.H. Hoang and Ch. Schwab. High-dimensional finite elements for elliptic problems with multiple scales. Multiscale Modeling \& Simulation, 3:168-194, 2005.

[7] W. E and B. Engquist. The heterogeneous multiscale methods. Commun. Math. Sci, 1:87-132, 2003.

[8] A. Abdulle, W. E, B. Engquist, and E. Vanden-Eijnden. The heterogeneous multiscale method. Acta Numer., 21:1-87, 2012.

[9] A. Abdulle and Ch. Schwab. Heterogeneous Multiscale FEM for Diffusion Problems on Rough Surfaces. SIAM Multiscale Modeling \& Simulation, 3(1):195-220, 2005.

[10] W. E, P Ming, and P. Zhang. Analysis of the heterogeneous multiscale method for elliptic homogenization problems. American Mathematical Society, 18(1):121-156, 2005.

[11] A. Abdulle. On A Priori Error Analysis of Fully Discrete Heterogeneous Multiscale FEM. SIAM Multiscale Modeling \& Simulation, 4(2):447-459, 2005.

[12] A. Abdulle. Heterogeneous multiscale method with quadrilateral elements. Numerical mathematics and advanced applications, Springer, Berlin, pages 743-751, 2006.

[13] A. Abdulle and B. Engquist. Finite element heterogeneous multiscale methods with near optimal computational complexity. SIAM Multiscale Modeling \& Simulation, 6(4):1059-1084, 2007.

[14] A. Abdulle. Multiscale method based on discontinuous Galerkin methods for homogenization problems. Comptes rendus-Mathématique, 346(1-2):97-102, 2008.

[15] Assyr Abdulle. Discontinuous galerkin finite element heterogeneous multiscale method for elliptic problems with multiple scales. Math. Comp., 81(278):687-713, 2012. 
[16] A. Abdulle. Analysis of a Heterogeneous Multiscale FEM for Problems in Elasticity. Math. Mod. Meth. Appl. Sci. (M3AS), 6:615-635, 2006.

[17] A. Abdulle. The finite element heterogeneous multiscale method: a computational strategy for multiscale PDEs. GAKUTO International Series, Math. Sci. Appl., 31:135-181, 2009.

[18] I. Babuška and W.C. Rheinboldt. Error estimates for adaptive finite element computations. SIAM Journal on Numerical Analysis, 15(4):736-754, 1978.

[19] M. Ainsworth and J.T. Oden. A posteriori error estimation in finite element analysis. Computer Methods in Applied Mechanics and Engineering, 142(1-2):1-88, 1997.

[20] R. Verfürth. A Review of A Posteriori Error Estimation $\&$ Adaptive Mesh-Refinement Techniques. Wiley-Teubner, 1996.

[21] I. Babuška and T. Strouboulis. The finite element method and its reliability. Oxford University Press, USA, 2001.

[22] S. Prudhomme and J.T. Oden. On goal-oriented error estimation for elliptic problems: application to the control of pointwise errors. Computer Methods in Applied Mechanics and Engineering, 176(14):313-331, 1999.

[23] J.T. Oden and S. Prudhomme. Goal-oriented error estimation and adaptivity for the finite element method. Computers $\&$ Mathematics with Applications, 41(5-6):735 - 756, 2001.

[24] M. B. Giles and E. Süli. Adjoint methods for PDEs: a posteriori error analysis and postprocessing by duality. Acta Numer., 11:145-236, 2002.

[25] W. Bangerth and R. Rannacher. Adaptive Finite Element Methods for Differential Equations. Birkhäuser Verlag, Basel, 2003.

[26] R. Becker and R. Rannacher. An optimal control approach to a posteriori error estimation in finite element methods. Acta numerica, 10:1-102, 2001.

[27] R.H. Nochetto, A. Veeser, and M. Verani. A safeguarded dual weighted residual method. IMA journal of Numerical Analysis, 29(1):126-140, 2009.

[28] M. Ainsworth and R. Rankin. Guaranteed computable bounds on quantities of interest in finite element computations. International Journal of Numerical Methods in Engineering, 89:1605-1634, 2012 .

[29] J.T. Oden, S. Prudhomme, A. Romkes, and P.T. Bauman. Multiscale modeling of physical phenomena: Adaptive control of models. SIAM Journal on Scientific Computing, 28(6):2359-2392, 2006 .

[30] T.J.R. Hughes. Multiscale phenomena: Green's functions, the Dirichlet-to-Neumann formulation, subgrid scale models, bubbles and the origins of stabilized methods* 1 . Computer methods in applied mechanics and engineering, 127(1-4):387-401, 1995.

[31] M.G. Larson and A. Målqvist. Adaptive variational multiscale methods based on a posteriori error estimation: Energy norm estimates for elliptic problems. Computer Methods in Applied Mechanics and Engineering, 196(21-24):2313-2324, 2007.

[32] J. Nolen, G. Papanicolaou, and O. Pironneau. A framework for adaptive multiscale methods for elliptic problems. Multiscale Model. Simul, 7(1):171-196, 2008.

[33] M. Ohlberger. A posteriori error estimates for the heterogeneous multiscale finite element method for elliptic homogenization problems. SIAM Multiscale Modeling ES Simulation, 4:88-114, 2005.

[34] P. Henning and M. Ohlberger. A-posteriori error estimation for a heterogeneous multiscale method for monotone operators and beyond a periodic setting. Preprint 01/11 - N, FB 10, Universität Münster, 2011.

[35] G. Nguetseng. A general convergence result for a functional related to the theory of homogenization. SIAM Journal on Mathematical Analysis, 20:608, 1989. 
[36] A. Abdulle and A. Nonnenmacher. A posteriori error analysis of the heterogeneous multiscale method for homogenization problems. Comptes Rendus Mathematique, 347(17-18):1081-1086, 2009.

[37] A. Abdulle and A. Nonnenmacher. Adaptive finite element heterogeneous multiscale method for homogenization problems. Computer Methods in Applied Mechanics and Engineering, 200:2710$2726,2011$.

[38] A. Bensoussan, J.L. Lions, and G. Papanicolaou. Asymptotic analysis for periodic structures (Studies in mathematics and its applications). Elsevier North-Holland, 1978.

[39] V. V. Jikov, S. M. Kozlov, and O. A. Oleinik. Homogenization of Differential Operators and Integral Functionals. Springer-Verlag Telos, 1994.

[40] E. De Giorgi and S. Spagnolo. Sulla convergenza degli integrali dell'energia per operatori ellittici del secondo ordine. Boll. Un. Mat. Ital, 8(4):391-411, 1973.

[41] François Murat and Luc Tartar. H-convergence, topics in the mathematical modeling of composite materials. Progr. Nonlinear Differential Equations Appl., 31:21-43, 1997.

[42] P. Ciarlet and P.-A. Raviart. The combined effect of curved boundaries and numerical integration in isoparametric finite element methods. Math. Foundation of the FEM with Applications to PDE, pages 409-474, 1972.

[43] A. Abdulle. A priori and a posteriori error analysis for numerical homogenization: a unified framework. Ser. Contemp. Appl. Math. CAM, 16:280-305, 2011.

[44] O.A. Ladyzhenskaya. Boundary value problems of mathematical physics. Springer-Verlag New York, 1985 .

[45] L. Bers, F. John, and M. Schechter. Partial differential equations. Lectures in Applied Mathematics, Proceedings of the Summer Seminar, Boulder, CO, 1957.

[46] E. Suli. Review of the book Adaptive finite element methods for differential equations by W. Bangerth and R. Rannacher. Math. Comp., 74:1033-1052, 2004.

[47] H.J. Schmid. On cubature formulae with a minimal number of knots. Numerische Mathematik, 31(3):281-297, 1978 .

[48] A. Nonnenmacher. Adaptive Finite Element Methods for Multiscale Partial Differential Equations. PhD thesis, EPFL, 2011.

[49] O. Zienkiewicz. The Finite Element Method. Elsevier/Butterworth-Heinemann, Amsterdam, 2005.

[50] R. Cools. Constructing cubature formulae: the science behind the art. Acta Numerica, 6:1-54, 1997.

[51] A. Abdulle and Y. Bai. A reduced basis heterogeneous multiscale method. submitted for publication, 2011.

[52] P.A. Raviart. The use of numerical integration in finite element methods for solving parabolic equations. Miller, J. J. H. (ed.), Topics in Numerical Analysis, Academic Press, pages 233-264, 1973.

[53] A. Abdulle and A. Nonnenmacher. A short and versatile finite element multiscale code for homogenization problems. Computer Methods in Applied Mechanics and Engineering, 198(37-40):2839-2859, 2009 .

[54] L. Chen. iFEM: an innovative finite element methods package in MATLAB. In Preparation, 2008.

[55] S. Bartels, C. Carstensen, and A. Hecht. P2Q2Iso2D=2D isoparametric FEM in Matlab. Journal of Computational and Applied Mathematics, 192(2):219-250, 2006.

[56] A. Abdulle and Y. Bai. Reduced basis finite element heterogeneous multiscale method for high-order discretizations of elliptic homogenization problems. J. Comput. Phys., in press, 2012. 